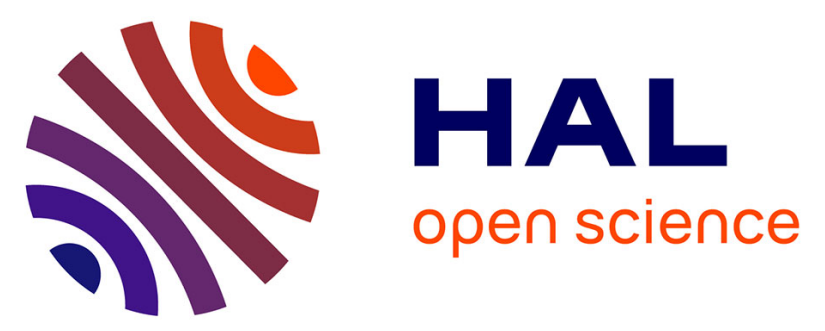

\title{
Dramatic influence of the substitution of alkylidene-5H-furan-2-ones in Diels-Alder cycloadditions with o-quinonedimethide as diene partner: en route to the CDEF polycyclic ring system of lactonamycin
} Sébastien Dubois, Fabien Rodier, Romain Blanc, Raphaël Rahmani, Virginie Héran, Jérôme Thibonnet, Laurent Commeiras, Jean-Luc Parrain

\section{To cite this version:}

Sébastien Dubois, Fabien Rodier, Romain Blanc, Raphaël Rahmani, Virginie Héran, et al.. Dramatic influence of the substitution of alkylidene-5H-furan-2-ones in Diels-Alder cycloadditions with o-quinonedimethide as diene partner: en route to the CDEF polycyclic ring system of lactonamycin. Organic \& Biomolecular Chemistry, 2012, 10, pp.4712-4719. 10.1039/c2ob25299f . hal-00736663

\section{HAL Id: hal-00736663 https://hal.science/hal-00736663}

Submitted on 28 Sep 2013

HAL is a multi-disciplinary open access archive for the deposit and dissemination of scientific research documents, whether they are published or not. The documents may come from teaching and research institutions in France or abroad, or from public or private research centers.
L'archive ouverte pluridisciplinaire HAL, est destinée au dépôt et à la diffusion de documents scientifiques de niveau recherche, publiés ou non, émanant des établissements d'enseignement et de recherche français ou étrangers, des laboratoires publics ou privés. 


\title{
Organic \& \\ Biomolecular \\ Chemistry
}

Cite this: Org. Biomol. Chem., 2012, 10,4712

wWw.rsc.org/obc

PAPER

\section{Dramatic influence of the substitution of alkylidene-5H-furan-2-ones in Diels-Alder cycloadditions with o-quinonedimethide as diene partner: en route to the CDEF polycyclic ring system of lactonamycin $\uparrow$}

\author{
Sébastien Dubois, ${ }^{a}$ Fabien Rodier, ${ }^{a}$ Romain Blanc, ${ }^{a}$ Raphaël Rahmani, ${ }^{a}$ Virginie Héran, ${ }^{a}$ Jérôme Thibonnet, ${ }^{b}$ \\ Laurent Commeiras* ${ }^{* a}$ and Jean-Luc Parrain*a
}

Received 10th February 2012, Accepted 12th April 2012

DOI: 10.1039/c2ob25299f

An efficient and rapid synthesis of the CDEF ring system of lactonamycinone is reported via a highly

chemo- and diastereoselective intermolecular Diels-Alder cycloaddition between trans-1,2-

disilyloxybenzocyclobutene and the appropriate $\gamma$-alkylidenebutenolide. The feasibility and the total

chemoselectivity of the [4+2] cycloaddition for the construction of a spirolactone moiety via an

intramolecular approach (IMDA) using both partners is also described demonstrating the versatility of the

$\gamma$-alkylidenebutenolide building block.

\section{Introduction}

Due to their intrinsic conformational features and their structural implications in biological systems, the preparation of polycyclic structures fused at a central carbon remains an important challenge in organic synthesis. ${ }^{1}$ The asymmetric character of the molecule brought by the stereogenic spiro carbon is generally responsible for the biological activities. In this context and over the last two decades, numerous natural spirolactones have been isolated from different sources and some of them exhibit impressive biological activities. Selected examples include abyssomicin $\mathrm{C}^{2}$ and lactonamycin, ${ }^{3}$ two potent antibacterial agents against Gram-positive bacteria, including resistant Staphylococcus aureus strains (Fig. 1).

From a structural point of view, lactonamycin presents a spirolactone moiety and a 2,3-dihydronaphthalene-1,4-dione core. The strategy usually used to access a 1,2,3,4-tetrahydronaphthalene substructure is an inter- or intramolecular $[4+2]$ reaction using benzocyclobutene derivatives as diene partners. ${ }^{4}$ Regarding the spirolactone part, most of the methods reported involve a prior installation of the tertiary alcohol, which is then followed by an intramolecular esterification. Numerous methods for the

\footnotetext{
${ }^{a}$ Aix-Marseille Université, Institut des Sciences Moléculaires de Marseille iSm2, UMR 7313, avenue Escadrille Normandie Niemen, 13397 Marseille cedex 20,France.E-mail: laurent.commeiras@ univ-amu.fr, jl.parrain@univ-amu.fr; Fax: (+33)-(0)491-288-861; Tel: (+33)-(0)491-289-126

${ }^{b}$ Université François Rabelais, Département de Chimie, Laboratoire PCMB, 32 Avenue Monge, 37200 Tours, France

$\dagger$ Electronic supplementary information (ESI) available: Experimental procedures and characterisation for all novel compounds. CCDC 849361. For ESI and crystallographic data in CIF or other electronic
} format see DOI: $10.1039 / \mathrm{c} 2 \mathrm{ob} 25299 \mathrm{f}$ construction of spirolactones with concomitant formation of the fused quaternary centre have also been reported. Notably, pericyclic reactions have demonstrated to be quite efficient in the synthesis of natural products. ${ }^{5}$ Moreover, we recently disclosed a highly chemo- and diastereoselective intermolecular Diels-Alder cycloaddition between trans-1,2-disilyloxybenzocyclobutene $\mathbf{1}$ and methylprotoanemonine 2 to access the lambertellol backbone 3 (Scheme 1). ${ }^{6}$ It transpired from this work that the chemoselectivity of such a transformation was highly dependent on the nature of the substituents onto the lactone. Accordingly, $\delta$-substituted alkylidenebutenolides $\mathbf{4}$ furnished the naphthofuranone moiety $\mathbf{5}$ whereas the $\alpha$-bromo- $\beta, \delta$-substituted lactones led to the corresponding spiro-cycloadducts $\mathbf{6}$. However, when the reaction was performed with lactone 7 , no cycloadduct was obtained and this is certainly due to both stereoelectronic and steric factors.

It is worth to note that $\gamma$-alkylidenebutenolides have been repeatedly used in Diels-Alder reactions, ${ }^{7-14}$ however we were the first one to realise a full study of the reactivity of the $\delta$-substituted ones in the intermolecular version. ${ }^{7 e, 9}$ In addition, different laboratories have developed their approach to the title natural product, ${ }^{15}$ however, only one total synthesis of lactonamycin ${ }^{16}$ and one total synthesis of its aglycone ${ }^{17}$ have been reported to
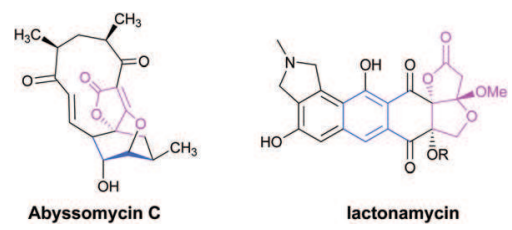

Fig. 1 Examples of natural products containing the spirolactone moiety. 
date. In connection with our interest in the total synthesis of natural product possessing interesting biological properties and in the development of new methodologies to efficiently prepare spirolactone moiety, ${ }^{18}$ we have undertaken the synthesis of the CDEF ring system of lactonamycinone via an intermolecular Diels-Alder reaction. An account of the intramolecular silicon tethered $[4+2]$ cyclisation between a benzocyclobutene and a $\gamma$-alkylidenebutenolide will also be given.

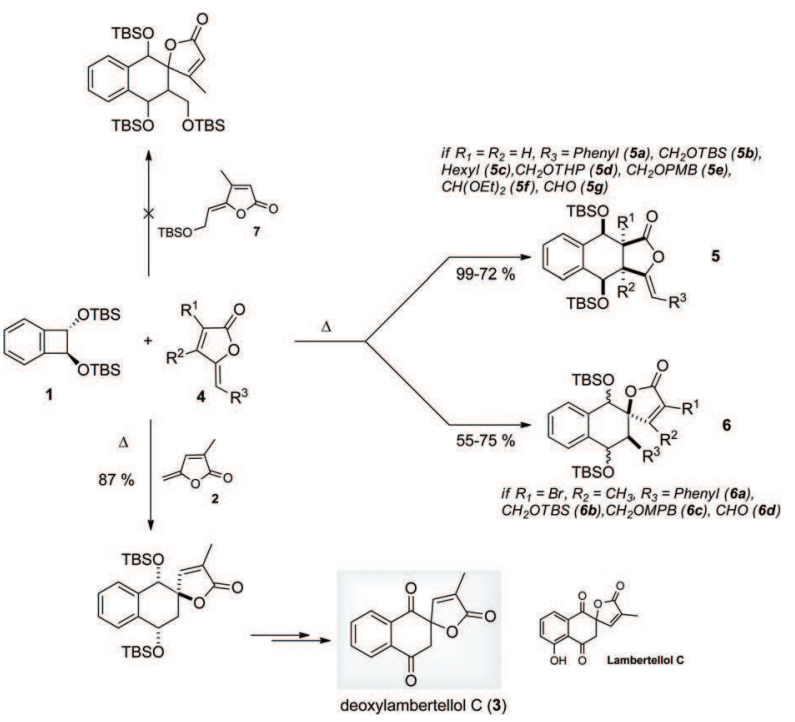

Scheme 1 Diels-Alder cycloaddition between trans-1,2-disilyloxybenzocyclobutene $\mathbf{1}$ and $\gamma$-alkylidenebutenolides.

\section{Results and discussion}

As outlined in Scheme 2, our retrosynthesis consists in two main disconnections to access the CDEF polycyclic ring system of lactonamycinone. Accordingly, a late stage intramolecular oxaMichael addition would allow the formation of the E ring. On the other hand, the CDF tricyclic spirolactone could efficiently be reached via an intermolecular [4+2] reaction between 1 and the appropriate lactone 8. Our program was started with confidence as our previous results suggested that substituents onto the $\alpha, \beta$-double bond of the alkylidenebutenolides prevented the cyclisation onto the endo-cyclic alkene and therefore, favoured reaction onto the exo-cyclic one. Having this in mind, and in connection with our approach toward the CDEF skeleton of lactonamycinone, we thought of using a $\gamma$-alkylidenebutenolide decorated with the electron donating methoxy group onto the $\beta$ position of the lactone. In order to validate this approach, lactone (Z)-8 was chosen as model substrate and was prepared in two steps from methyltetronate $\mathbf{9}^{19}$ and aldehyde $\mathbf{1 0}$ (Scheme 3). ${ }^{20}$ Addition of the homoenolate of $\mathbf{9}$ onto aldehyde $\mathbf{1 0}$ furnished alcohol 11 in 74\% yield as an inseparable $52: 48$ mixture of diastereomers. Then, compound $\mathbf{1 1}$ underwent a dehydration to afford a separable 67:33 mixture of $\gamma$-alkylidenebutenolides (Z)-8 and (E)-8 respectively, in 56\% yield. As for the dienophilic benzocyclobutene partner $\mathbf{1}$, it was synthesised on a multigram scale according to methods described by South and Liebeskind ${ }^{21}$ and Danishefsky et $a l^{22}$ Having the two precursors in hand, we were now ready for the key cyclisation step. Under the conditions previously reported for the $\alpha$-bromo- $\beta, \delta$-substituted lactone (i.e. $50{ }^{\circ} \mathrm{C}, 4 \mathrm{~h}$ in degassed benzene), the cycloaddition
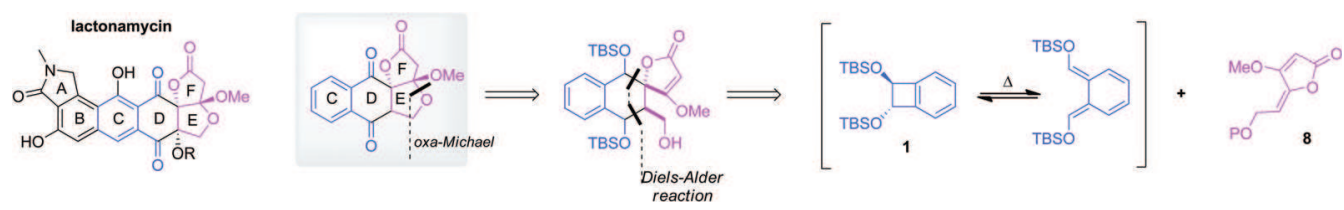

Scheme 2 Retrosynthetic analysis.

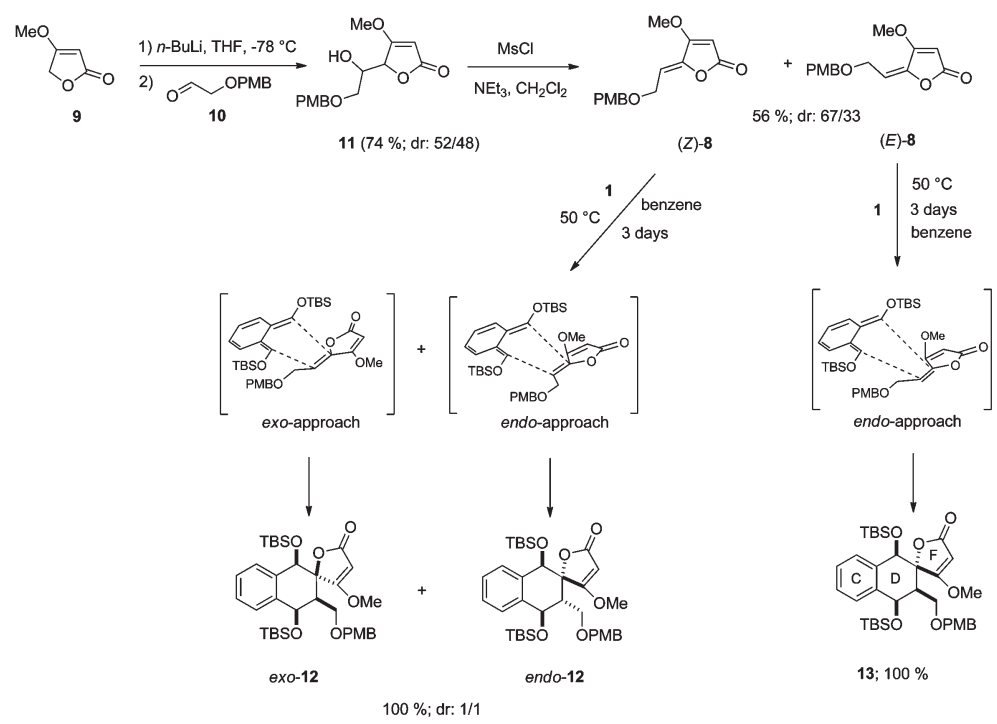

Scheme 3 Studies on the synthesis of the CDF ring system of lactonamycinone. 


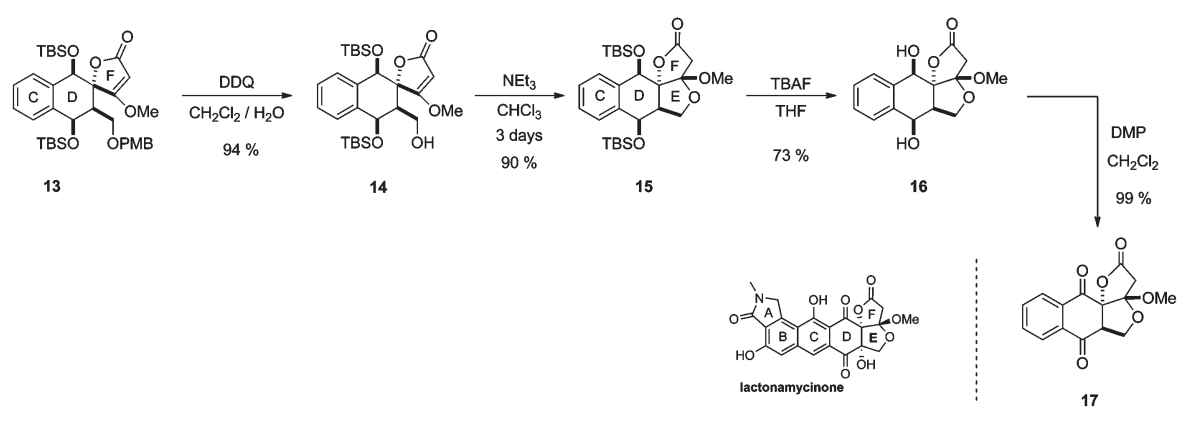

Scheme 4 Synthesis of the CDEF ring system of lactonamycinone.

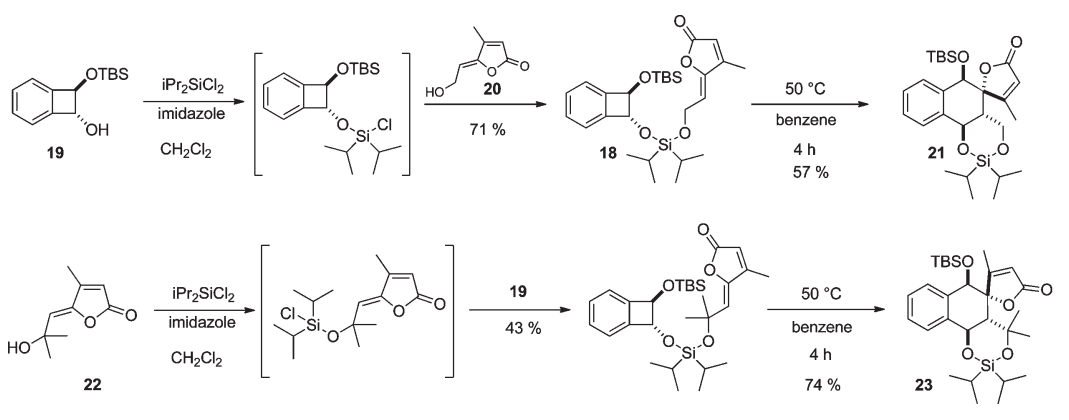

Scheme 5 Intramolecular approach.

only furnished a very small amount (up to $5 \%$ ) of the expected spirolactone 12. Starting materials 1 and (Z)-8 were recovered unchanged at the end of the reaction. Gratifyingly, when the reaction time was prolonged from four hours to three days, ${ }^{23}$ the Diels-Alder reaction gave the desired spiro-cycloadduct $\mathbf{1 2}$ in a quantitative manner and as a $1: 1$ mixture of diastereomers arising from an endo- and an exo-approach respectively.

The feasibility of the intermolecular cycloaddition leading to the desired spirolactone moiety thus validated, we next turn our attention to the $(E)$-lactone 8. Accordingly, the hetero Michael reaction to form the E-ring required the pending primary alcohol onto compound 14 being cis to the single carbon-carbon bond of the lactone. The only way to access such a cycloadduct was to start with the $(E)$-double bond onto lactone 8. Pleasingly, the cycloaddition reaction was not only efficient and provided the expected compound $\mathbf{1 3}$ in a quantitative manner, but was also totally diastereoselective in favour of the endo-approach. Thereafter, an additional four steps were performed to access the CDEF rings of lactonamicynone (Scheme 4). Deprotection of the PMB group using DDQ in wet $\mathrm{CH}_{2} \mathrm{Cl}_{2}$ afforded primary alcohol 14 in 94\% yield.

The latter underwent the intramolecular oxa-Michael addition under basic conditions to form the last E-ring $\mathbf{1 5}$ in $90 \%$ yield and as a single diastereomer. Following which, a deprotection of the bis silylether $\mathbf{1 5}$ in presence of tetrabutylammonium fluoride gave the corresponding diol 16 in $73 \%$ yield. Finally, the sensitive quinone $\mathbf{1 7}$ was obtained after oxidation of the diol in the presence of an excess of Dess-Martin periodinane (10 equiv) in a quantitative manner. To summarise, the tetracyclic skeleton of lactonamycinone 17 was diastereoselectively synthesised in $62 \%$ yield over five steps from lactone $(E)-\mathbf{8}$. It goes without saying that the method developed here is a powerful and competitive tool to access rapidly and efficiently the scaffold of natural products containing spirolactone moieties.

As mentioned earlier in the introduction, the $\delta$-substituted $\alpha, \beta$-unsubstituted alkylidenebutenolides failed to furnish the desired spirolactones and led instead to the corresponding naphthofuranone derivatives $\mathbf{5}$. In addition, lactone 7 proved to be unreactive during this intermolecular [4+2] process. In order to overturn the limits encountered and thus, to have access to a range of analogs of the lactonamycine core, we envisioned an intermolecular version of the previous strategy using a disposable silicon tether between the two cycloaddition partners. According to numerous reports, such approaches are favoured for entropic reasons and often lead to better chemo-, regio- and stereoselectivities. $^{23}$

The synthesis of the temporary silaketal tether derivative $\mathbf{1 8}$ required the prior preparation of alcohols 19 and 20. Monoprotected diol 19 (Scheme 5) was obtained in two steps from benzocyclobutanedione. Bis reduction of the ketones in the presence of $\mathrm{NaBH}_{4}$ in $\mathrm{MeOH}$ followed by treatment of the corresponding crude diol in the presence of one equivalent of $\mathrm{TBSCl}$ furnished the desired compound 19 in yields ranging from 33 to $76 \%$ yield over two steps. Both alcohols 19 and $\mathbf{2 0}^{24}$ were then linked together by sequential reaction with $\mathrm{iPr}_{2} \mathrm{SiCl}_{2}$ (Scheme 5). ${ }^{25}$ Gratifyingly, silaketal $\mathbf{1 8}$ was obtained in $71 \%$ yield. The silicon tethered molecule $\mathbf{1 8}$ was then heated at $55{ }^{\circ} \mathrm{C}$ for $4 \mathrm{~h}$ in benzene. Unlike its intermolecular counterpart (Scheme 1; lactone 7), silaketal 18 smoothly underwent the [4 +2] cyclisation in a chemo-, regio- and diastereoselective manner. The desired tetracyclic spirolactone $\mathbf{2 1}$ was pleasingly obtained in $57 \%$ yield. We then demonstrated the compatibility of the strategy with the more hindered lactone 22 (Scheme 5). The cycloadduct 23 was obtained in 74\% yield with total control of the 
chemo-, regio- and diastereoselectivity. The relative configurations of $\mathbf{2 1}$ and $\mathbf{2 3}$ were unambiguously established based on NOESY NMR experiments and through X-ray crystallographic analysis of $\mathbf{2 3}^{26}$ thus, confirming the endo-approach of the intramolecular cycloaddition reaction. Furthermore, we have shown that the chemoselectivity of the intermolecular approach could be overturned thanks to the silylated tether. Accordingly, when the benzocyclobutene is linked with an $\alpha, \beta$-unsubstituted lactone the sole product isolated resulted from the reaction onto the exocyclic double bond of the lactone. No naphthofuranone 5 was observed and the desired spirolactone $\mathbf{2 5}$ was obtained in $28 \%$ isolated yield over two steps. While $10 \%$ of the cycloadduct arising from the $E$-isomer of the starting lactone $\mathbf{2 4}$ was observed in the crude ${ }^{1} \mathrm{H}$ NMR spectrum, none was observed after purification on silica gel. This is probably due to its instability and might explain why the diastereomer $\mathbf{2 5}$ was isolated as the sole product and in modest yield.

\section{Conclusion}

In summary, a new highly diastereoselective and convergent approach towards the CDEF ring system of lactonamycinone has been reported. The key intermolecular Diels-Alder reaction between trans-1,2-disiloxybenzocyclobutene and the appropriate $\gamma$-alkylidenebutenolide allowed the concomitant formation of the fused spiro carbon together with the creation of three tertiary stereocentres with total control of the diastereoselectivity and in only 5 steps. In addition, an intramolecular cycloaddition using a disposable silicon tether to reach the desired spirolactone moiety has also been developed when its intermolecular counterpart failed to give the desired spiro-cycloadduct (i.e. when $\delta$ - or $\beta, \delta$-substituted were used).

\section{Experimental section}

\section{Compounds $(Z)-8$ and $(E)-8$}

To a stirred solution of THF at $-80{ }^{\circ} \mathrm{C}$ was added a solution of n-BuLi (7.7 mL, $193 \mathrm{mmol}, 1.1$ equiv, $2.5 \mathrm{M}$ in hexane). A precooled solution of methyltetronate $9^{19}$ ( 2 g, 175 mmol, 1 equiv) in THF $(35 \mathrm{~mL})$ was then added dropwise at $-80{ }^{\circ} \mathrm{C}$. After $20 \mathrm{~min}$ at $-80^{\circ} \mathrm{C}$, a precooled solution of aldehyde $\mathbf{1 0}^{20}(3.4 \mathrm{~g}$, $175 \mathrm{mmol}, 1$ equiv) in THF (17 mL) was added to the mixture and allowed to warm at room temperature. After 2 hours, ice crush followed by diluted aqueous $\mathrm{HCl}$ were added to the mixture. The aqueous phase was extracted with $\mathrm{Et}_{2} \mathrm{O}$. The combined organic phases were dried over $\mathrm{Na}_{2} \mathrm{SO}_{4}$ and concentrated under vacuum. The crude product was then purified by flash chromatography (3:7 petroleum ether-ethyl acetate) to afford a inseparable $52: 48$ mixture of alcohol 11 (3.2 g) in 74\% yield. To a stirred solution of alcohol 11 (1.14 g, $3.87 \mathrm{mmol}, 1$ equiv) in $\mathrm{CH}_{2} \mathrm{Cl}_{2}(7 \mathrm{~mL})$, was added triethylamine $(1.61 \mathrm{~mL}$, $11.6 \mathrm{mmol}, 3$ equiv) followed by mesylchloride $(0.419 \mathrm{~mL}$, $5.43 \mathrm{mmol}, 1.4$ equiv) were added dropwise. The mixture was heated at reflux overnight. The reaction was then quenched by addition of saturated aqueous solution of $\mathrm{NH}_{4} \mathrm{Cl}$. The aqueous phase was extracted with $\mathrm{CH}_{2} \mathrm{Cl}_{2}$. The combined organic phases were dried over $\mathrm{Na}_{2} \mathrm{SO}_{4}$ and concentrated under vacuum. The crude material was finally purified by flash chromatography
(8:2 petroleum ether-ether) to afford (598 mg, 56\% yield) (Z)-8 $(404 \mathrm{mg})$ and $(E)-8(194 \mathrm{mg})$ in $67 / 33$ ratio. $(Z)-8: \delta_{\mathrm{H}}$ $\left(400 \mathrm{MHz}, \mathrm{CDCl}_{3}\right) 3.81\left(3 \mathrm{H}, \mathrm{CH}_{3}\right), 3.92\left(3 \mathrm{H}, \mathrm{CH}_{3}\right), 4.30(2 \mathrm{H}$, $\left.\mathrm{d}, J=6.8 \mathrm{~Hz}, \mathrm{CH}_{2}\right), 4.46\left(2 \mathrm{H}, \mathrm{s}, \mathrm{CH}_{2}\right), 5.24(1 \mathrm{H}$, br s, $\mathrm{CH})$, $5.59(1 \mathrm{H}, \mathrm{t}, J=6.8 \mathrm{~Hz}, \mathrm{CH}), 6.88\left(2 \mathrm{H}, \mathrm{d}, J=8.5,2 \times \mathrm{CH}_{\mathrm{Ar}}\right)$, $7.27\left(2 \mathrm{H}, \mathrm{d}, J=8.5 \mathrm{~Hz}, 2 \times \mathrm{CH}_{\mathrm{Ar}}\right) ; \delta_{\mathrm{C}}\left(75 \mathrm{MHz}, \mathrm{CDCl}_{3}\right) 55.4$ $\left(\mathrm{CH}_{3}\right), 59.3\left(\mathrm{CH}_{3}\right), 63.7\left(\mathrm{CH}_{2}\right), 72.7\left(\mathrm{CH}_{2}\right), 89.8(\mathrm{CH}), 106.5$ $(\mathrm{CH}), 114.0\left(2 \times \mathrm{CH}_{\mathrm{Ar}}\right), 129.7\left(2 \times \mathrm{CH}_{\mathrm{Ar}}\right), 130.0(\mathrm{C}), 144.6(\mathrm{C})$, 159.5 (C), 168.2 (C), 169.9 (C); HRMS found 277.1074 [M + $\mathrm{H}]^{+}, \mathrm{C}_{15} \mathrm{H}_{17} \mathrm{O}_{5}$ requires 277.1071. (E)-8: $\delta_{\mathrm{H}}\left(400 \mathrm{MHz}, \mathrm{CDCl}_{3}\right)$ $3.80\left(3 \mathrm{H}, \mathrm{s}, \mathrm{CH}_{3}\right), 3,88\left(3 \mathrm{H}, \mathrm{s}, \mathrm{CH}_{3}\right), 4.39\left(2 \mathrm{H}, \mathrm{d}, J=7.6, \mathrm{CH}_{2}\right)$, $4.46\left(2 \mathrm{H}, \mathrm{s}, 1 \mathrm{H}, \mathrm{CH}_{2}\right), 5.30(1 \mathrm{H}$, br d, $J=1.3 \mathrm{~Hz}, \mathrm{CH}), 5.84$ $(1 \mathrm{H}, \mathrm{dt}, J=7.6$ and $1.3 \mathrm{~Hz}, \mathrm{CH}), 6.88(2 \mathrm{H}, \mathrm{d}, J=8.7 \mathrm{~Hz}, 2 \times$ $\left.\mathrm{CH}_{\mathrm{Ar}}\right), 7.27\left(2 \mathrm{H}, \mathrm{d}, J=8.7 \mathrm{~Hz}, 2 \times \mathrm{CH}_{\mathrm{Ar}}\right) ; \delta_{\mathrm{C}}(100 \mathrm{MHz}$, $\left.\mathrm{CDCl}_{3}\right) 55.4\left(\mathrm{CH}_{3}\right), 59.5\left(\mathrm{CH}_{3}\right), 63.2\left(\mathrm{CH}_{2}\right), 72.2\left(\mathrm{CH}_{2}\right), 91.8$ $(\mathrm{CH}), 111.8(\mathrm{CH}), 113.9\left(2 \times \mathrm{CH}_{\mathrm{Ar}}\right), 129.6\left(2 \times \mathrm{CH}_{\mathrm{Ar}}\right), 130.0$ (C), 144.4 (C), 159.5 (C), 167.9 (C), 170.5 (C); HRMS found $277.1074[\mathrm{M}+\mathrm{H}]^{+}, \mathrm{C}_{15} \mathrm{H}_{17} \mathrm{O}_{5}$ requires 277.1071.

\section{Compound 12}

In a oven-dried Schlenk tube, trans-1,2-bis(tert-butyldimethylsilyloxy)-1,2-benzocyclobutene $\mathbf{1}^{21,22}$ (326 mg, $0.89 \mathrm{mmol}, 1.5$ equiv) and butenolide ( $Z$ )-8 (164 mg, $0.59 \mathrm{mmol}, 1$ equiv) were dissolved in benzene- $\mathrm{D}_{6}(3.3 \mathrm{~mL})$. The solution was degassed for $10 \mathrm{~min}$ at $-80{ }^{\circ} \mathrm{C}$ three times. The mixture was then heated at $55{ }^{\circ} \mathrm{C}$. The reaction was followed by ${ }^{1} \mathrm{H}$ NMR and after disappearance of ( $Z$ )-8 (3 days), the solvent was removed under vacuum. The crude product was purified by flash chromatography ( $8: 2$ petroleum ether-ethyl acetate) to give a separable 1: 1 mixture (374 mg, 100\%) of exo-12 and endo-12. endo-12: $\delta_{\mathrm{H}}\left(400 \mathrm{MHz}, \mathrm{C}_{6} \mathrm{D}_{6}\right) 0.03\left(6 \mathrm{H}, \mathrm{s}, 2 \times \mathrm{CH}_{3}\right), 0.04\left(3 \mathrm{H}, \mathrm{s}, \mathrm{CH}_{3}\right)$, $0.37\left(3 \mathrm{H}, \mathrm{s}, \mathrm{CH}_{3}\right), 1.00\left(9 \mathrm{H}, \mathrm{s}, 3 \times \mathrm{CH}_{3}\right), 1.04\left(9 \mathrm{H}, \mathrm{s}, 3 \times \mathrm{CH}_{3}\right)$, 2.52-2.58 (1H, m, CH), $2.71\left(3 \mathrm{H}, \mathrm{s}, \mathrm{CH}_{3}\right), 3.32\left(3 \mathrm{H}, \mathrm{s}, \mathrm{CH}_{3}\right)$, $3.81\left(3 \mathrm{H}, \mathrm{dd}, J=9\right.$ and $\left.3.0 \mathrm{~Hz}, \mathrm{CH}_{2}\right), 3.89-3.94\left(1 \mathrm{H}, \mathrm{m}, \mathrm{CH}_{2}\right)$, $4.40\left(1 \mathrm{H}, \mathrm{d}, \mathrm{CH}_{2}, J=11.1 \mathrm{~Hz}\right), 4.49\left(1 \mathrm{H}, \mathrm{d}, \mathrm{CH}_{2}, J=11.1 \mathrm{~Hz}\right)$, $4.75(1 \mathrm{H}, \mathrm{s}, \mathrm{C}), 5.00(1 \mathrm{H}, \mathrm{d}, J=10 \mathrm{~Hz}, \mathrm{CH}), 5.15(1 \mathrm{H}, \mathrm{s}, \mathrm{CH})$, $6.84\left(2 \mathrm{H}, \mathrm{d}, J=8.7 \mathrm{~Hz}, 2 \times \mathrm{CH}_{\mathrm{Ar}}\right), 7.21-7.28(2 \mathrm{H}, \mathrm{m}, 2 \times$ $\left.\mathrm{CH}_{\mathrm{Ar}}\right), 7.35\left(2 \mathrm{H}, \mathrm{d}, J=8.7 \mathrm{~Hz}, 2 \times \mathrm{CH}_{\mathrm{Ar}}\right), 7.62-7.63(1 \mathrm{H}, \mathrm{m}$, $\left.\mathrm{CH}_{\mathrm{Ar}}\right), 7.68-7.70\left(1 \mathrm{H}, \mathrm{m}, \mathrm{CH}_{\mathrm{Ar}}\right) ; \delta_{\mathrm{C}}\left(100 \mathrm{MHz}, \mathrm{C}_{6} \mathrm{D}_{6}\right)-4.7$ $\left(\mathrm{CH}_{3}\right),-4.6\left(2 \times \mathrm{CH}_{3}\right),-4.1\left(\mathrm{CH}_{3}\right), 18.4(\mathrm{C}), 18.5(\mathrm{C}), 26.1$ (3 $\left.\times \mathrm{CH}_{3}\right), 26.2\left(3 \times \mathrm{CH}_{3}\right), 48.6(\mathrm{CH}), 54.8\left(\mathrm{CH}_{3}\right), 58.3\left(\mathrm{CH}_{3}\right)$, $67.1\left(\mathrm{CH}_{2}\right), 67.5(\mathrm{CH}), 72.1(\mathrm{CH}), 73.5\left(\mathrm{CH}_{2}\right), 86.6(\mathrm{C}), 90.3$ $(\mathrm{CH}), 114.1\left(2 \times \mathrm{CH}_{\mathrm{Ar}}\right), 123.5(\mathrm{CH}), 124.2(\mathrm{CH}), 127.1(\mathrm{CH})$, $127.4(\mathrm{CH}), 130.2\left(2 \times \mathrm{CH}_{\mathrm{Ar}}\right), 130.9(\mathrm{C}), 135.6(\mathrm{C}), 140.1(\mathrm{C})$, 159.9 (C), 171.6 (C), $182.4(\mathrm{C})$; IR $\left(v_{\max } / \mathrm{cm}^{-1}\right): 2956,2930$, 2889, 2856, 1759, 1637, 1613, 1510, 1460, 1366, 1247, 1173, 1131, 1098, 1070, $1036 \mathrm{~cm}^{-1}$; MS: $m / z(\mathrm{ESI}+) 663(\mathrm{M}+\mathrm{Na})^{+}$; HRMS found $658.3588\left[\mathrm{M}+\mathrm{NH}_{4}\right]^{+}, \mathrm{C}_{35} \mathrm{H}_{56} \mathrm{NO}_{7} \mathrm{Si}_{2}$ requires 658.3590. exo-12: $\delta_{\mathrm{H}}\left(400 \mathrm{MHz}, \mathrm{C}_{6} \mathrm{D}_{6}\right)-0.12\left(3 \mathrm{H}, \mathrm{s}, \mathrm{CH}_{3}\right)$, $-0.01\left(3 \mathrm{H}, \mathrm{s}, \mathrm{CH}_{3}\right), 0.06\left(3 \mathrm{H}, \mathrm{s}, \mathrm{CH}_{3}\right), 0.12\left(3 \mathrm{H}, \mathrm{s}, \mathrm{CH}_{3}\right), 0.99$ $\left(9 \mathrm{H}, \mathrm{s}, 3 \times \mathrm{CH}_{3}\right), 1.04\left(9 \mathrm{H}, \mathrm{s}, 3 \times \mathrm{CH}_{3}\right), 3.02\left(3 \mathrm{H}, \mathrm{s}, \mathrm{CH}_{3}\right)$, 3.11-3.16 (1H, m, CH), 3.25-3.32 (2H, m, $\left.\mathrm{CH}_{2}\right), 3.27(3 \mathrm{H}, \mathrm{s}$, $\left.\mathrm{CH}_{3}\right), 3.98\left(1 \mathrm{H}, \mathrm{dd}, J=8.9\right.$ and $\left.3 \mathrm{~Hz}, \mathrm{CH}_{2}\right), 4.08\left(1 \mathrm{H}, \mathrm{d}, \mathrm{CH}_{2}, J\right.$ $=11 \mathrm{~Hz}, \mathrm{H} 9), 4.38\left(1 \mathrm{H}, \mathrm{d}, \mathrm{CH}_{2}, J=11 \mathrm{~Hz}\right), 4.82(1 \mathrm{H}, \mathrm{s}, \mathrm{CH})$, $4.94(1 \mathrm{H}, \mathrm{s}, \mathrm{CH}), 5.11(1 \mathrm{H}, \mathrm{d}, J=6.8 \mathrm{~Hz}, \mathrm{CH}), 6.75(2 \mathrm{H}, \mathrm{d}, J=$ $\left.8.7 \mathrm{~Hz}, 2 \times \mathrm{CH}_{\mathrm{Ar}}\right), 7.20\left(2 \mathrm{H}, \mathrm{d}, J=8.7 \mathrm{~Hz}, 2 \times \mathrm{CH}_{\mathrm{Ar}}\right)$, 7.26-7.37 (2H, m, $\left.\mathrm{CH}_{\mathrm{Ar}}\right), 7.64\left(1 \mathrm{H}\right.$, br d, $\left.J=6.8 \mathrm{~Hz}, \mathrm{CH}_{\mathrm{Ar}}\right)$, 
$7.77\left(1 \mathrm{H}\right.$, br d, $\left.J=7.5 \mathrm{~Hz}, \mathrm{CH}_{\mathrm{Ar}}\right) ; \delta_{\mathrm{C}}\left(100 \mathrm{MHz}, \mathrm{C}_{6} \mathrm{D}_{6}\right)-5.5$ $\left(\mathrm{CH}_{3}\right),-5.0\left(\mathrm{CH}_{3}\right),-4.8\left(\mathrm{CH}_{3}\right),-4.3\left(\mathrm{CH}_{3}\right), 18.4(\mathrm{C}), 18.6(\mathrm{C})$, $26.0\left(6 \times \mathrm{CH}_{3}\right), 47.3(\mathrm{CH}), 54.7\left(\mathrm{CH}_{3}\right), 58.5\left(\mathrm{CH}_{3}\right), 66.1\left(\mathrm{CH}_{2}\right)$, $67.6(\mathrm{CH}), 68.7(\mathrm{CH}), 73.4\left(\mathrm{CH}_{2}\right), 86.0(\mathrm{C}), 91.0(\mathrm{CH}), 113.9(2$ $\left.\times \mathrm{CH}_{\mathrm{Ar}}\right), 122.8(\mathrm{CH}), 124.7(\mathrm{CH}), 127.5(\mathrm{CH}), 127.6(\mathrm{CH})$, $130.2\left(2 \times \mathrm{CH}_{\mathrm{Ar}}\right), 131.1(\mathrm{C}), 136.0(\mathrm{C}), 137.9$ (C), $159.6(\mathrm{C})$, 171.1 (C), 180.8 (C, C2); IR $\left(v_{\max } / \mathrm{cm}^{-1}\right) 2952,2930,2888$, 2857, 1756, 1641, 1515, 1471, 1461, 1360, 1247, 1192, 1171, 1131, 1071, 1031; MS: $m / z(E S I+) 663(\mathrm{M}+\mathrm{Na})^{+}$; HRMS found $658.3589\left[\mathrm{M}+\mathrm{NH}_{4}\right]^{+}, \mathrm{C}_{35} \mathrm{H}_{56} \mathrm{NO}_{7} \mathrm{Si}_{2}$ requires 658.3590 .

\section{Compound endo-13}

In a oven-dried Schlenk tube, trans-1,2-bis(tert-butyldimethylsilyloxy)-1,2-benzocyclobutene 1 ( $253 \mathrm{mg}, 0.69 \mathrm{mmol}, 1.5$ equiv) and butenolide $(E)-8$ (128 mg, $0.46 \mathrm{mmol}, 1$ equiv) were dissolved in benzene- $\mathrm{d}_{6}(2.6 \mathrm{~mL})$. The solution was degassed for $10 \mathrm{~min}$ at $-80{ }^{\circ} \mathrm{C}$ three times. The mixture was then heated at $55{ }^{\circ} \mathrm{C}$. The reaction was followed by ${ }^{1} \mathrm{H}$ NMR and after disappearance of $(E)-8$ (3 days), the solvent was removed under vacuum. The crude product was purified by flash chromatography (9:1 petroleum ether-ethyl acetate) to give endo-13 (269 mg, 100\%). $\delta_{\mathrm{H}}\left(400 \mathrm{MHz}, \mathrm{C}_{6} \mathrm{D}_{6}\right) 0.03\left(3 \mathrm{H}, \mathrm{s}, \mathrm{CH}_{3}\right), 0.05$ $\left(3 \mathrm{H}, \mathrm{s}, \mathrm{CH}_{3}\right), 0.6\left(3 \mathrm{H}, \mathrm{s}, \mathrm{CH}_{3}\right), 0.34\left(3 \mathrm{H}, \mathrm{s}, \mathrm{CH}_{3}\right), 0.95(9 \mathrm{H}, \mathrm{s}, 3$ $\left.\times \mathrm{CH}_{3}\right), 1.04\left(9 \mathrm{H}, \mathrm{s}, 3 \times \mathrm{CH}_{3}\right), 2.9\left(3 \mathrm{H}, \mathrm{s}, \mathrm{CH}_{3}\right), 3.01-3.06(1 \mathrm{H}$, $\left.\mathrm{m}, \mathrm{CH}_{2}\right), 3.11-3.16(1 \mathrm{H}, \mathrm{m}, \mathrm{CH}), 3.30\left(3 \mathrm{H}, \mathrm{s}, \mathrm{CH}_{3}\right), 3.88(1 \mathrm{H}$, dd, $J=9.8$ and $\left.3.5 \mathrm{~Hz}, \mathrm{CH}_{2}\right), 4.02\left(1 \mathrm{H}, \mathrm{d}, \mathrm{CH}_{2}, J=11.8 \mathrm{~Hz}\right)$, $4.07\left(1 \mathrm{H}, \mathrm{d}, \mathrm{CH}_{2}, J=11.8 \mathrm{~Hz}\right), 4.86(1 \mathrm{H}, \mathrm{s}, \mathrm{CH}), 5.24(1 \mathrm{H}, \mathrm{br}$ s, CH), $5.32(1 \mathrm{H}, \mathrm{d}, J=5.3 \mathrm{~Hz}, \mathrm{CH}), 6.76(2 \mathrm{H}, \mathrm{d}, J=8.5 \mathrm{~Hz}, 2$ $\left.\times \mathrm{CH}_{\mathrm{Ar}}\right), 7.07\left(2 \mathrm{H}, \mathrm{d}, J=8.5 \mathrm{~Hz}, 2 \times \mathrm{CH}_{\mathrm{Ar}}\right), 7.20-7.27(2 \mathrm{H}, \mathrm{m}$, $\left.2 \times \mathrm{CH}_{\mathrm{Ar}}\right), 7.66\left(1 \mathrm{H}\right.$, br d, $\left.J=5.5 \mathrm{~Hz}, \mathrm{CH}_{\mathrm{Ar}}\right), 7.72(1 \mathrm{H}, \mathrm{br} \mathrm{d}, J$ $\left.=5.8 \mathrm{~Hz}, \mathrm{CH}_{\mathrm{Ar}}\right) ; \delta_{\mathrm{H}}\left(400 \mathrm{MHz}, \mathrm{CDCl}_{3}\right) 0.07\left(3 \mathrm{H}, \mathrm{s}, \mathrm{CH}_{3}\right), 0.17$ $\left(3 \mathrm{H}, \mathrm{s}, \mathrm{CH}_{3}\right), 0.18\left(3 \mathrm{H}, \mathrm{s}, \mathrm{CH}_{3}\right), 0.19\left(3 \mathrm{H}, \mathrm{s}, \mathrm{CH}_{3}\right), 0.96(9 \mathrm{H}, \mathrm{s}$, $\left.3 \times \mathrm{CH}_{3}\right), 0.98\left(9 \mathrm{H}, \mathrm{s}, 3 \times \mathrm{CH}_{3}\right), 2.69-2.76\left(1 \mathrm{H}, \mathrm{m}, \mathrm{CH}_{2}\right)$, 2.81-2.87 (1H, m, CH), $3.52\left(3 \mathrm{H}, \mathrm{s}, \mathrm{CH}_{3}\right), 3.65(1 \mathrm{H}, \mathrm{dd}, J=9.6$ and $\left.2.8 \mathrm{~Hz}, \mathrm{CH}_{2}\right), 4.15\left(3 \mathrm{H}, \mathrm{s}, \mathrm{CH}_{3}\right), 4.15\left(2 \mathrm{H}, \mathrm{s}, \mathrm{CH}_{2}\right), 4.96$ $(1 \mathrm{H}$, br s, CH), $4.98(1 \mathrm{H}$, br s, CH), $5.08(1 \mathrm{H}, \mathrm{d}, J=5.1 \mathrm{~Hz}$, $\mathrm{CH}), 6.80\left(2 \mathrm{H}, \mathrm{d}, J=8.5 \mathrm{~Hz}, 2 \times \mathrm{CH}_{\mathrm{Ar}}\right), 7.08(2 \mathrm{H}, \mathrm{d}, J=8.5$ $\left.\mathrm{Hz}, 2 \times \mathrm{CH}_{\mathrm{Ar}}\right), 7.29-7.32\left(2 \mathrm{H}, \mathrm{m}, 2 \times \mathrm{CH}_{\mathrm{Ar}}\right), 7.40-7.46(2 \mathrm{H}, \mathrm{m}$, $\left.2 \times \mathrm{CH}_{\mathrm{Ar}}\right) ; \delta\left(75 \mathrm{MHz}, \mathrm{CDCl}_{3}\right) \delta-5.0\left(\mathrm{CH}_{3}\right),-4.9\left(\mathrm{CH}_{3}\right),-4.8$ $\left(\mathrm{CH}_{3}\right),-4.7\left(\mathrm{CH}_{3}\right), 18.1(\mathrm{C}), 18.2(\mathrm{C}), 25.8\left(3 \times \mathrm{CH}_{3}\right), 25.9(3$ $\left.\times \mathrm{CH}_{3}\right), 52.0(\mathrm{CH}), 55.2\left(\mathrm{CH}_{3}\right), 58.8\left(\mathrm{CH}_{3}\right), 64.7\left(\mathrm{CH}_{2}\right), 66.6$ $(\mathrm{CH}), 71.6(\mathrm{CH}), 72.1\left(\mathrm{CH}_{2}\right), 88.1(\mathrm{C}), 90.0(\mathrm{CH}), 113.6(2 \times$ $\left.\mathrm{CH}_{\mathrm{Ar}}\right), 122.3(\mathrm{CH}), 123.1(\mathrm{CH}), 126.7(\mathrm{CH}), 126.8(\mathrm{CH}), 128.5$ $\left(2 \times \mathrm{CH}_{\mathrm{Ar}}\right), 130.4(\mathrm{C}), 134.6(\mathrm{C}), 138.3(\mathrm{C}), 158.9$ (C), 172.3 (C), 182.9 (C); MS: $m / z(\mathrm{ESI}+) 664(\mathrm{M}+\mathrm{Na})^{+}$; HRMS found $641.3327[\mathrm{M}+\mathrm{H}]^{+}, \mathrm{C}_{35} \mathrm{H}_{53} \mathrm{O}_{7} \mathrm{Si}_{2}$ requires 641.3324 .

\section{Compound 14}

To a stirred solution of endo-13 (260 mg, $0.405 \mathrm{mmol}, 1$ equiv) in $10.5 \mathrm{~mL}$ of $5 \%$ aqueous $\mathrm{CH}_{2} \mathrm{Cl}_{2}$ was added at $0{ }^{\circ} \mathrm{C}$ DDQ (102 mg, $0.446 \mathrm{mmol}, 1.1$ equiv). After 1 hour at $0{ }^{\circ} \mathrm{C}$ the solution was stirred at room temperature until disappearance of starting material (1 hour) then filtered through a pad of florosil and celite then concentrated. The crude product was purified by flash chromatography $(8: 2$ petroleum ether-ethyl acetate) to give $14(199 \mathrm{mg}, 94 \%)$. mp $209^{\circ} \mathrm{C} ; \delta_{\mathrm{H}}\left(400 \mathrm{MHz}, \mathrm{CDCl}_{3}\right) 0.07$ $\left(3 \mathrm{H}, \mathrm{s}, \mathrm{CH}_{3}\right), 0.17\left(3 \mathrm{H}, \mathrm{s}, \mathrm{CH}_{3}\right), 0.19\left(3 \mathrm{H}, \mathrm{s}, \mathrm{CH}_{3}\right), 0.23(3 \mathrm{H}, \mathrm{s}$, $\left.\mathrm{CH}_{3}\right), 0.96\left(9 \mathrm{H}, \mathrm{s}, 3 \times \mathrm{CH}_{3}\right), 1.02\left(9 \mathrm{H}, \mathrm{s}, 3 \times \mathrm{CH}_{3}\right), 2.67(1 \mathrm{H}$, $\mathrm{m}, \mathrm{OH}), 2.82(1 \mathrm{H}, \mathrm{dt}, J=8.3$ and $5.5 \mathrm{~Hz}, \mathrm{CH}), 3.18(1 \mathrm{H}, \mathrm{dd}, J$ $=11.6$ and $\left.4.8 \mathrm{~Hz}, \mathrm{CH}_{2}\right), 3.50(1 \mathrm{H}, \mathrm{dd}, J=11.6$ and $8.3 \mathrm{~Hz}$, $\left.\mathrm{CH}_{2}\right), 3.58\left(3 \mathrm{H}, \mathrm{s}, \mathrm{CH}_{3}\right), 4.89(1 \mathrm{H}, \mathrm{s}, \mathrm{CH}), 5.03(1 \mathrm{H}, \mathrm{s}, \mathrm{CH})$, $5.21(1 \mathrm{H}, \mathrm{d}, \mathrm{CH}, J=5.5 \mathrm{~Hz}, \mathrm{H} 7), 7.30-7.37\left(2 \mathrm{H}, \mathrm{m}, 2 \times \mathrm{CH}_{\mathrm{Ar}}\right)$, 7.41-7.48 (2H, m, $\left.2 \times \mathrm{CH}_{\mathrm{Ar}}\right) ; \delta_{\mathrm{C}}\left(75 \mathrm{MHz}, \mathrm{CDCl}_{3}\right)-5.0\left(\mathrm{CH}_{3}\right)$, $-4.93\left(\mathrm{CH}_{3}\right),-4.87\left(\mathrm{CH}_{3}\right),-4.8\left(\mathrm{CH}_{3}\right), 18.1(\mathrm{C}), 18.3(\mathrm{C}), 25.9$ $\left(6 \times \mathrm{CH}_{3}\right), 52.8(\mathrm{CH}), 58.9\left(\mathrm{CH}_{3}\right), 60.6\left(\mathrm{CH}_{2}\right), 69.1(\mathrm{CH}), 71.3$ $(\mathrm{CH}), 88.6(\mathrm{C}), 90.6(\mathrm{CH}), 122.8\left(\mathrm{CH}_{\mathrm{Ar}}\right), 123.5\left(\mathrm{CH}_{\mathrm{Ar}}\right), 126.9$ $\left(\mathrm{CH}_{\mathrm{Ar}}\right), 127.0\left(\mathrm{CH}_{\mathrm{Ar}}\right), 134.3\left(\mathrm{C}_{\mathrm{Ar}}\right), 137.5\left(\mathrm{C}_{\mathrm{Ar}}\right), 171.9(\mathrm{C})$, 181.9 (C); IR $\left(v_{\max } / \mathrm{cm}^{-1}\right) 3451,2952,2929,2888,2857,1738$, $1628,1471,1459,1252,1185,1130,1068,1049$; MS: $\mathrm{m} / \mathrm{z}$ $(\mathrm{ESI}+) 543(\mathrm{M}+\mathrm{Na})^{+}$; HRMS found $521.2747[\mathrm{M}+\mathrm{H}]^{+}$, $\mathrm{C}_{27} \mathrm{H}_{45} \mathrm{O}_{6} \mathrm{Si}_{2}$ requires 521.2749.

\section{Compound 15}

A solution of 14 (145 mg, $0.279 \mathrm{mmol}, 1$ equiv), $\mathrm{NEt}_{3}(116 \mu \mathrm{L}$, $0.837 \mathrm{mmol}, 3$ equiv) in $\mathrm{CHCl}_{3}(15 \mathrm{~mL})$ was stirred at room temperature. After disappearance of the starting material (3 days), the solvent was removed under vacuum and the crude product was purified by flash chromatography $(9: 1$ petroleum ether-ethyl acetate) to afford $15(131 \mathrm{mg}, 90 \%) . \delta_{\mathrm{H}}(400 \mathrm{MHz}$, $\left.\mathrm{CDCl}_{3}\right) 0.09\left(3 \mathrm{H}, \mathrm{s}, \mathrm{CH}_{3}\right), 0.17\left(3 \mathrm{H}, \mathrm{s}, \mathrm{CH}_{3}\right), 0.19\left(3 \mathrm{H}, \mathrm{s}, \mathrm{CH}_{3}\right)$, $0.20\left(3 \mathrm{H}, \mathrm{s}, \mathrm{CH}_{3}\right), 0.99\left(9 \mathrm{H}, \mathrm{s}, 3 \times \mathrm{CH}_{3}\right), 1.03\left(9 \mathrm{H}, \mathrm{s}, 3 \times \mathrm{CH}_{3}\right)$, $2.75\left(1 \mathrm{H}, \mathrm{d}, J=16.8 \mathrm{~Hz}, \mathrm{CH}_{2}\right), 2.81\left(1 \mathrm{H}, \mathrm{d}, J=16.8 \mathrm{~Hz}, \mathrm{CH}_{2}\right)$, $2.88\left(3 \mathrm{H}, \mathrm{s}, \mathrm{CH}_{3}\right), 3.13-3.19(1 \mathrm{H}, \mathrm{m}, \mathrm{CH}), 3.30-3.35(1 \mathrm{H}, \mathrm{m}$, $\left.\mathrm{CH}_{2}\right), 4.03-4.08\left(1 \mathrm{H}, \mathrm{m}, \mathrm{CH}_{2}\right), 4.94(1 \mathrm{H}$, br s, $\mathrm{CH}), 5.02(1 \mathrm{H}$, $\mathrm{d}, J=6.5 \mathrm{~Hz}, \mathrm{CH}), 7.26-7.39\left(2 \mathrm{H}, \mathrm{m}, 2 \times \mathrm{CH}_{\mathrm{Ar}}\right), 7.39-7.46$ $\left(2 \mathrm{H}, \mathrm{m}, 2 \times \mathrm{CH}_{\mathrm{Ar}}\right), \delta_{\mathrm{C}}\left(75 \mathrm{MHz}, \mathrm{CDCl}_{3}\right)-4.9\left(\mathrm{CH}_{3}\right),-4.8_{3}$ $\left(\mathrm{CH}_{3}\right),-4.77\left(\mathrm{CH}_{3}\right),-4.6\left(\mathrm{CH}_{3}\right), 18.3(2 \times \mathrm{C}), 25.9\left(3 \times \mathrm{CH}_{3}\right)$, $26.2\left(3 \times \mathrm{CH}_{3}\right), 39.1\left(\mathrm{CH}_{2}\right), 50.5\left(\mathrm{CH}_{3}\right), 51.5(\mathrm{CH}), 67.1(\mathrm{CH})$, $68.8\left(\mathrm{CH}_{2}\right), 70.8(\mathrm{CH}), 95.9(\mathrm{C}), 112.3(\mathrm{C}), 123.2\left(\mathrm{CH}_{\mathrm{Ar}}\right), 123.7$ $\left(\mathrm{CH}_{\mathrm{Ar}}\right), 126.3\left(\mathrm{CH}_{\mathrm{Ar}}\right), 126.6\left(\mathrm{CH}_{\mathrm{Ar}}\right), 135.6\left(\mathrm{C}_{\mathrm{Ar}}\right), 136.2\left(\mathrm{C}_{\mathrm{Ar}}\right)$, 172.6 (C); IR $\left(v_{\max } / \mathrm{cm}^{-1}\right) 2954,2930,2888,2857,1791,1472$, 1461, 1251, 1211, 1182, 1126, 1076, 1061, 1013; MS: $\mathrm{m} / \mathrm{z}$ $(\mathrm{ESI}+) 543(\mathrm{M}+\mathrm{Na})^{+}$; HRMS found $538.3011\left[\mathrm{M}+\mathrm{NH}_{4}\right]^{+}$, $\mathrm{C}_{27} \mathrm{H}_{48} \mathrm{NO}_{6} \mathrm{Si}_{2}$ requires 538.3015.

\section{Compound 16}

In an oven-dried flask, 15 (130 mg, $0.25 \mathrm{mmol}, 1$ equiv) was dissolved in THF $(8 \mathrm{~mL})$. At $0{ }^{\circ} \mathrm{C}$, a TBAF solution $(0.625 \mathrm{~mL}$, $0.625 \mathrm{mmol}, 1 \mathrm{M}$ in THF, 2.5 equiv) was added dropwise. The solution was stirred at room temperature and after completion of the reaction (1 hour), the mixture was quenched with aqueous saturated $\mathrm{NaHCO}_{3}$ solution. The aqueous layer was extracted with EtOAc. The combined organic phases were dried over $\mathrm{Na}_{2} \mathrm{SO}_{4}$ then concentrated under vacuum. The crude product was purified by flash chromatography $(1: 1$ petroleum etherethyl acetate) to give the diol $16(53 \mathrm{mg}, 73 \%) . \mathrm{mp} 251{ }^{\circ} \mathrm{C} ; \delta_{\mathrm{H}}$ $\left(400 \mathrm{MHz}, \mathrm{CDCl}_{3}\right) 2.87\left(1 \mathrm{H}, \mathrm{d}, J=17.6 \mathrm{~Hz}, \mathrm{CH}_{2}\right), 2.96(1 \mathrm{H}, \mathrm{d}$, $\left.J=17.6 \mathrm{~Hz}, \mathrm{CH}_{2}\right), 3.16\left(3 \mathrm{H}, \mathrm{s}, \mathrm{CH}_{3}\right), 3.18-3.21(1 \mathrm{H}, \mathrm{m}, \mathrm{CH})$, $3.46(1 \mathrm{H}$, br d, $J=8.3 \mathrm{~Hz}, \mathrm{OH}), 3.51-3.56\left(1 \mathrm{H}, \mathrm{m}, \mathrm{CH}_{2}\right)$, 4.23-4.27 $\left(1 \mathrm{H}, \mathrm{m}, \mathrm{CH}_{2}\right), 4.88(1 \mathrm{H}, \mathrm{br} \mathrm{d}, J=8.3 \mathrm{~Hz}) 5.07(1 \mathrm{H}$, $\mathrm{d}, J=6.3 \mathrm{~Hz}, \mathrm{CH}), 7.36-7.38\left(2 \mathrm{H}, \mathrm{m}, 2 \times \mathrm{CH}_{\mathrm{Ar}}\right), 7.48-7.52$ $\left(2 \mathrm{H}, \mathrm{m}, 2 \times \mathrm{CH}_{\mathrm{Ar}}\right) ; \delta_{\mathrm{C}}\left(75 \mathrm{MHz}, \mathrm{CDCl}_{3} / \mathrm{MeOD}\right) 38.8\left(\mathrm{CH}_{2}\right)$, 
$49.8\left(\mathrm{CH}_{3}\right), 50.8(\mathrm{CH}), 65.1(\mathrm{CH}), 68.5\left(\mathrm{CH}_{2}\right), 69.6(\mathrm{CH}), 96.5$ (C), $112.0(\mathrm{C}), 122.2\left(\mathrm{CH}_{\mathrm{Ar}}\right), 122.5\left(\mathrm{CH}_{\mathrm{Ar}}\right), 126.0\left(\mathrm{CH}_{\mathrm{Ar}}\right)$, $126.4\left(\mathrm{CH}_{\mathrm{Ar}}\right), 135.2\left(\mathrm{C}_{\mathrm{Ar}}\right), 135.7\left(\mathrm{C}_{\mathrm{Ar}}\right), 173.5(\mathrm{C})$; IR $\left(v_{\max } /\right.$ $\mathrm{cm}^{-1}$ ) 3484, 3411, 2988, 2934, 2892, 1768, 1458, 1412, 1281, 1262, 1249, 1228, 1188, 1140, 1099, 1063, 1048, 1033, 1007; MS: $m / z(\mathrm{ESI}+) 315(\mathrm{M}+\mathrm{Na})^{+}$; HRMS found 293.1019 $[\mathrm{M}+\mathrm{H}]^{+}, \mathrm{C}_{15} \mathrm{H}_{17} \mathrm{O}_{6}$ requires 293.1020.

\section{Compound 17}

To a solution of diol 16 (16 mg, $0.055 \mathrm{mmol}, 1$ equiv) in $\mathrm{CH}_{2} \mathrm{Cl}_{2}(7 \mathrm{~mL})$, under argon, at $0{ }^{\circ} \mathrm{C}$, was added Dess-Martin periodinane $(232 \mathrm{mg}, 0.55 \mathrm{mmol}, 10$ equiv). The reaction mixture was stirred at room temperature and monitored by TLC. After disappearance of the starting material, the mixture was poured into (1/1) mixture of saturated aqueous solution of $\mathrm{Na}_{2} \mathrm{~S}_{2} \mathrm{O}_{3}$ and saturated aqueous solution of $\mathrm{NaHCO}_{3}(25 \mathrm{~mL})$ and shaken vigorously for $5 \mathrm{~min}$. The aqueous layer was extracted with $\mathrm{CH}_{2} \mathrm{Cl}_{2}$. The combined organic layers were washed with a saturated aqueous $\mathrm{NaHCO}_{3}$ solution, saturated aqueous $\mathrm{NaCl}$, dried over $\mathrm{Na}_{2} \mathrm{SO}_{4}$, dried over $\mathrm{Na}_{2} \mathrm{SO}_{4}$ and concentrated under vacuum to give the crude product $17(16 \mathrm{mg}$, $100 \%) . \delta_{\mathrm{H}}\left(400 \mathrm{MHz}, \mathrm{CDCl}_{3}\right) 2.92\left(1 \mathrm{H}, \mathrm{d}, J=16.8 \mathrm{~Hz}, \mathrm{CH}_{2}\right)$, $2.99\left(1 \mathrm{H}, \mathrm{d}, J=16.8 \mathrm{~Hz}, \mathrm{CH}_{2}\right), 3.17\left(3 \mathrm{H}, \mathrm{s}, \mathrm{CH}_{3}\right), 3.80(1 \mathrm{H}, \mathrm{dd}$, $J=8.0$ and $3.8 \mathrm{~Hz}, \mathrm{CH}), 4.54\left(1 \mathrm{H}, \mathrm{m}, \mathrm{CH}_{2}\right), 4.75(1 \mathrm{H}, \mathrm{dd}, J=$ 9.0 and $\left.3.8 \mathrm{~Hz}, \mathrm{CH}_{2}\right), 7.80-7.85\left(2 \mathrm{H}, \mathrm{m}, 2 \times \mathrm{CH}_{\mathrm{Ar}}\right), 8.14-8.21$ $\left(2 \mathrm{H}, \mathrm{m}, 2 \times \mathrm{CH}_{\mathrm{Ar}}\right) ; \delta_{\mathrm{C}}\left(75 \mathrm{MHz}, \mathrm{CDCl}_{3}\right) 36.6\left(\mathrm{CH}_{2}\right), 52.6(\mathrm{CH})$, $53.7\left(\mathrm{CH}_{3}\right), 70.4\left(\mathrm{CH}_{2}\right), 90.7(\mathrm{C}), 113.4(\mathrm{C}), 127.4\left(\mathrm{CH}_{\mathrm{Ar}}\right)$, $127.7\left(\mathrm{CH}_{\mathrm{Ar}}\right), 134.2\left(\mathrm{C}_{\mathrm{Ar}}\right), 135.0\left(\mathrm{CH}_{\mathrm{Ar}}\right), 135.3\left(\mathrm{C}_{\mathrm{Ar}}\right), 135.4$ $\left(\mathrm{CH}_{\mathrm{Ar}}\right), 171.4(\mathrm{C}), 189.3(\mathrm{C}), 191.9(\mathrm{C})$; IR $\left(v_{\max } / \mathrm{cm}^{-1}\right) 2958$, 2919, 2850, 1730, 1711, 1668, 1641, 1591, 1563, 1437, 1340, $1328,1306,1258,1245,1175,1140,1087,1015$; HRMS found $306.0971\left[\mathrm{M}+\mathrm{NH}_{4}\right]^{+}, \mathrm{C}_{15} \mathrm{H}_{16} \mathrm{NO}_{6}$ requires 306.0972.

\section{Compound 19}

To a stirred solution of diketone ${ }^{21}(500 \mathrm{mg}, 3.78 \mathrm{mmol})$ in methanol $(50 \mathrm{~mL})$ at $0{ }^{\circ} \mathrm{C}$ was added sodium borohydride $(143 \mathrm{mg}, 3.78 \mathrm{mmol})$ portionwise $(10 \mathrm{mg}$ per $10 \mathrm{~min})$. After 1 hour, the solvent was removed under vacuum at $0{ }^{\circ} \mathrm{C}$. The crude product $(370 \mathrm{mg})$ was dissolved in DCM $(0.1 \mathrm{M})$ and cooled to $0{ }^{\circ} \mathrm{C}$. Imidazole $(157 \mathrm{mg}, 2.312 \mathrm{mmol}, 0.85$ equiv) was added to the mixture and TBSCl $(369 \mathrm{mg}, 2.45 \mathrm{mmol}, 0.9$ equiv) dissolved in $20 \mathrm{~mL}$ of DCM was added via a seringe pump $\left(3.6 \mathrm{~mL} \mathrm{~h}^{-1}\right)$. After one night at $0{ }^{\circ} \mathrm{C}$, the reaction was quenched by adding water. The aqueous layer was extracted with DCM and the organic phases were dried over $\mathrm{Na}_{2} \mathrm{SO}_{4}$ and the solvent removed under high vacuum. The crude product was purified by flash chromatography $\left(7: 3: \mathrm{EP}-\mathrm{Et}_{2} \mathrm{O}\right)$ to give the monoprotected benzocyclobutenediol 19 in variable amount $(33-76 \%) . \delta_{\mathrm{H}}\left(300 \mathrm{MHz}, \mathrm{CDCl}_{3}\right) 0.2\left(6 \mathrm{H}, \mathrm{s}, 2 \times \mathrm{CH}_{3}\right), 0.97$ $\left(9 \mathrm{H}, \mathrm{s}, 3 \times \mathrm{CH}_{3}\right), 2.33(1 \mathrm{H}, \mathrm{OH}), 4.92(1 \mathrm{H}, \mathrm{s}, \mathrm{CH}), 4.93(1 \mathrm{H}, \mathrm{s}$, $\mathrm{CH}), 7.27-7.36\left(4 \mathrm{H}, \mathrm{m}, 4 \times \mathrm{CH}_{\mathrm{Ar}}\right) ; \delta_{\mathrm{C}}\left(75 \mathrm{MHz}, \mathrm{CDCl}_{3}\right)-4.5(2$ $\left.\times \mathrm{CH}_{3}\right), 18.4(\mathrm{C}), 26.0\left(3 \times \mathrm{CH}_{3}\right), 79.9(2 \times \mathrm{CH}), 123.4(2 \times$ $\left.\mathrm{CH}_{\mathrm{Ar}}\right), 129.6\left(\mathrm{CH}_{\mathrm{Ar}}\right), 129.9\left(\mathrm{CH}_{\mathrm{Ar}}\right), 143.3\left(\mathrm{C}_{\mathrm{Ar}}\right), 144.3\left(\mathrm{C}_{\mathrm{Ar}}\right)$; MS: $m / z 273[\mathrm{M}+\mathrm{Na}]^{+}$; HRMS found $251.1462[\mathrm{M}+\mathrm{H}]^{+}$, $\mathrm{C}_{14} \mathrm{H}_{23} \mathrm{O}_{2} \mathrm{Si}$ requires 251.1462 .

\section{Compound 20}

To a stirred solution of the $\gamma$-butenolide ${ }^{27}(400 \mathrm{mg}, 1.57 \mathrm{mmol}$, 1 equiv) in anhydrous THF $(8.2 \mathrm{~mL})$ at $0{ }^{\circ} \mathrm{C}$ was added a solution of HF-pyridine $(121 \mu \mathrm{L}, 70 \%$ in pyridine, $4.72 \mathrm{mmol}, 3$ equiv). The reaction was stirred at room temperature and followed by TLC. After disappearance of the starting material, the reaction was quenched with a saturated aqueous solution of $\mathrm{NaHCO}_{3}$. The aqueous phase was extracted with ether and the combined organics layers were dried over anhydrous $\mathrm{MgSO}_{4}$, filtered and concentrated in vacuo. The resulting crude product was purified by flash chromatography on silica gel (petroleum ether-ethyl acetate $1: 1)$ to give $20(157 \mathrm{mg}, 71 \%) . \delta_{\mathrm{H}}$ $\left(400 \mathrm{MHz}, \mathrm{C}_{6} \mathrm{D}_{6}\right) 1.27\left(3 \mathrm{H}, \mathrm{d}, J=1.3 \mathrm{~Hz}, \mathrm{CH}_{3}\right), 4.23(2 \mathrm{H}, \mathrm{d}, J$ $\left.=6.8 \mathrm{~Hz}, \mathrm{CH}_{2}\right), 4.94(1 \mathrm{H}, \mathrm{CH}, \mathrm{td}, J=6.8$ and $0.8 \mathrm{~Hz}, \mathrm{CH}), 5.32$ $(1 \mathrm{H}, \mathrm{m}, \mathrm{CH}) ; \delta_{\mathrm{C}}\left(100 \mathrm{MHz}, \mathrm{C}_{6} \mathrm{D}_{6}\right) 10.9\left(\mathrm{CH}_{3}\right), 57.3\left(\mathrm{CH}_{2}\right)$, $110.5(\mathrm{CH}), 117.1(\mathrm{CH}), 150.4(\mathrm{C}), 154.6(\mathrm{C}), 168.5(\mathrm{C})$; MS: $\mathrm{m} / \mathrm{z}(\mathrm{ESI}+) 163(\mathrm{M}+\mathrm{Na})^{+}$.

\section{Compound 21}

To a stirred solution of $\mathrm{CH}_{2} \mathrm{Cl}_{2}(0.6 \mathrm{~mL})$, imidazole $(38 \mathrm{mg}$, $0.56 \mathrm{mmol}, 5$ equiv) and $\mathrm{iPr}_{2} \mathrm{SiCl}_{2}(20 \mu \mathrm{L}, 0.11 \mathrm{mmol}, 1$ equiv) was added dropwise and at room temperature $19(28 \mathrm{mg}$, $0.11 \mathrm{mmol}, 1$ equiv) in $\mathrm{CH}_{2} \mathrm{Cl}_{2}(0.35 \mathrm{~mL})$. After disappearance of 20 (5 min), lactone 20 (16 $\mathrm{mg}, 0.11 \mathrm{mmol}, 1$ equiv) was added to the mixture. The reaction was stirred for $15 \mathrm{~min}$, then quenched with a saturated aqueous solution of $\mathrm{NH}_{4} \mathrm{Cl}$. The aqueous layer was extracted with $\mathrm{CH}_{2} \mathrm{Cl}_{2}$. The combined organic phase were dried over $\mathrm{Na}_{2} \mathrm{SO}_{4}$ and concentrated under vacuum. The crude product was purified by flash chromatography (petroleum ether-diethylether $85: 15)$ to give $\mathbf{1 8}(40 \mathrm{mg}, 71 \%)$. $\delta_{\mathrm{H}}$ $\left(300 \mathrm{MHz}, \mathrm{CDCl}_{3}\right) 0.19\left(6 \mathrm{H}, \mathrm{s}, 2 \times \mathrm{CH}_{3}\right), 0.94(9 \mathrm{H}, \mathrm{s}, 2 \times$ $\left.\mathrm{CH}_{3}\right), 1.02-1.06\left(3 \mathrm{H}, \mathrm{m}, \mathrm{CH}_{3}\right), 1.09-1.16\left(11 \mathrm{H}, \mathrm{m}, 3 \times \mathrm{CH}_{3}\right.$ and $\mathrm{CH}), 2.10\left(3 \mathrm{H}, \mathrm{br}, \mathrm{CH}_{3}\right), 4.69\left(2 \mathrm{H}, \mathrm{d}, J=6.3 \mathrm{~Hz}, \mathrm{CH}_{2}\right)$, $5.00(1 \mathrm{H}$, br s, CH $), 5.11\left(1 \mathrm{H}\right.$, br s, $\left.\mathrm{CH}_{3}\right), 5.40(1 \mathrm{H}, \mathrm{t}, J=6.3$ $\mathrm{Hz}, \mathrm{CH}), 5.93\left(1 \mathrm{H}\right.$, br s, $\left.\mathrm{CH}_{3}\right), 7.24-7.34\left(4 \mathrm{H}, \mathrm{m}, \mathrm{CH}_{\mathrm{Ar}}\right)$; $\mathrm{MS}$ : $m / z(\mathrm{ESI}+) 525[\mathrm{M}+\mathrm{Na}]^{+}$.

The ${ }^{1} \mathrm{H}$ NMR revealed the formation of the cycloadduct $\mathbf{1 8}$. Thus, no more characterisation was made on this kind of product.

In Schlenk tube, $18(40 \mathrm{mg})$ was heated $\left(55^{\circ} \mathrm{C}\right)$ in degassed $\mathrm{C}_{6} \mathrm{D}_{6}(2 \mathrm{~mL})$ for 4 hours. The solution was then concentrated and purified by flash chromatography (petroleum ether-diethylether $85: 15)$ to give $21(23 \mathrm{mg}, 57 \%) . \delta_{\mathrm{H}}\left(400 \mathrm{MHz}, \mathrm{C}_{6} \mathrm{D}_{6}\right)$ $-0.03\left(3 \mathrm{H}, \mathrm{s}, \mathrm{CH}_{3}\right), 0.20\left(3 \mathrm{H}, \mathrm{s}, \mathrm{CH}_{3}\right), 0.96\left(9 \mathrm{H}, \mathrm{s}, 3 \times \mathrm{CH}_{3}\right)$, $1.06\left(3 \mathrm{H}\right.$, br s, $\left.\mathrm{CH}_{3}\right), 1.08-1.13\left(14 \mathrm{H}, \mathrm{m}, 4 \times \mathrm{CH}_{3}\right.$ and $\left.2 \times \mathrm{CH}\right)$, $1.96(1 \mathrm{H}, \mathrm{td}, J=10.5$ and $3.5 \mathrm{~Hz}, \mathrm{CH}), 3.87(1 \mathrm{H}, \mathrm{dd}, J=11.5$ and $\left.3.5 \mathrm{~Hz}, \mathrm{CH}_{2}\right), 4.40\left(1 \mathrm{H}, \mathrm{t}, J=11.5 \mathrm{~Hz}, \mathrm{CH}_{2}\right), 4.91(1 \mathrm{H}, \mathrm{s}$, $\mathrm{CH}), 5.13(1 \mathrm{H}, \mathrm{d}, J=10.5 \mathrm{~Hz}, \mathrm{CH}), 5.33(1 \mathrm{H}, \mathrm{br} \mathrm{s}, \mathrm{CH})$, 7.17-7.27 (2H, m, $\left.2 \times \mathrm{CH}_{\mathrm{Ar}}\right), 7.55\left(1 \mathrm{H}, \mathrm{d}, J=7.5 \mathrm{~Hz}, \mathrm{CH}_{\mathrm{Ar}}\right)$, $7.84\left(1 \mathrm{H}, \mathrm{d}, J=7.5 \mathrm{~Hz}, \mathrm{CH}_{\mathrm{Ar}}\right) ; \delta_{\mathrm{C}}\left(100 \mathrm{MHz}, \mathrm{CDCl}_{3}\right) \delta=-4.8$ $\left(\mathrm{CH}_{3}\right),-4.7\left(\mathrm{CH}_{3}\right), 12.4\left(\mathrm{CH}_{3}\right), 13.5(2 \times \mathrm{CH}), 16.9\left(\mathrm{CH}_{3}\right), 17.0$ $\left(\mathrm{CH}_{3}\right), 17.2\left(\mathrm{CH}_{3}\right), 17.3\left(\mathrm{CH}_{3}\right), 18.4(\mathrm{C}), 21.1\left(3 \times \mathrm{CH}_{3}\right), 47.7$ $(\mathrm{CH}), 63.4\left(\mathrm{CH}_{2}\right), 71.2(\mathrm{CH}), 71.7(\mathrm{CH}), 77.8(\mathrm{CH}), 90.9(\mathrm{C})$, $118.5(\mathrm{CH}), 122.7(\mathrm{CH}), 123.5(\mathrm{CH}), 127.4(\mathrm{CH}), 127.8(\mathrm{CH})$, 127.9 (CH), 134.7 (C), 140.1 (C), 168.3 (C), 171.0 (C); IR $\left(v_{\max } / \mathrm{cm}^{-1}\right)$ 2953, 2928, 2861, 1771, 1463, 1261, 1133, 1071; MS $m / z(\mathrm{ESI}+) 525[\mathrm{M}+\mathrm{Na}]^{+}$; HRMS found 520.2907 $\left[\mathrm{M}+\mathrm{NH}_{4}{ }^{+}\right], \mathrm{C}_{27} \mathrm{H}_{46} \mathrm{NO}_{5} \mathrm{Si}_{2}$ requires 520.2909 . 


\section{Compound 22}

A dry Schlenk tube equipped with a Teflon-coated magnetic stirrer was charged with anhydrous $\mathrm{K}_{2} \mathrm{CO}_{3}$ (1.3 g, $9.40 \mathrm{mmol}, 2$ equiv) and ( $Z$ )- $\alpha, \beta$-insaturated- $\beta$-iodide acid ( $1 \mathrm{~g}, 4.70 \mathrm{mmol}, 1$ equiv). The mixture vessel was evacuated and backfilled with argon. Then freshly distilled DMF $(15 \mathrm{~mL})$ was added and the suspension was stirred for $15 \mathrm{~min}$ at room temperature. The mixture was degassed at $0{ }^{\circ} \mathrm{C}$ for $5 \mathrm{~min}$ and backfilled with argon. After reaching room temperature, the alkyne $(0.461 \mathrm{~g}$, $4.70 \mathrm{mmol}, 1$ equiv) and $\mathrm{CuI}(0.9 \mathrm{~g}, 4.70 \mathrm{mmol}, 1$ equiv) were added. The Schlenk tube was sealed and then placed in a preheated oil bath at $55^{\circ} \mathrm{C}$. Stirring was allowed for 4 hours. Then, the mixture was placed in an ice bath and a saturated aqueous solution of $\mathrm{NH}_{4} \mathrm{Cl}$ was added. Stirring at $0{ }^{\circ} \mathrm{C}$ was allowed for $10 \mathrm{~min}$ at which time the reactional mixture was diluted with ether and filtered through a short pad of Celite. The filtrate was washed with brine and the organic layer was dried over anhydrous $\mathrm{MgSO}_{4}$, filtered and concentrated in vacuo to yield the expecting $\gamma$-butyrolactone 22 (700 $\mathrm{mg}, 88 \%$ yield) which was engaged in the next step without further purifications. $\delta_{\mathrm{H}}$ (400 MHz, $\left.\mathrm{C}_{6} \mathrm{D}_{6}\right) 1.20\left(3 \mathrm{H}\right.$, br d, $\left.J=1.0 \mathrm{~Hz}, \mathrm{CH}_{3}\right), 1.36(6 \mathrm{H}, \mathrm{s}$, $\left.2 \times \mathrm{CH}_{3}\right), 5.02(1 \mathrm{H}, \mathrm{s}, \mathrm{CH}), 5.24(1 \mathrm{H}, \mathrm{m}, \mathrm{CH}) ; \delta_{\mathrm{C}}(100 \mathrm{MHz}$, $\left.\mathrm{C}_{6} \mathrm{D}_{6}\right) 11.0\left(\mathrm{CH}_{3}\right), 30.2\left(2 \times \mathrm{CH}_{3}\right), 70.3(\mathrm{C}), 116.0(\mathrm{CH}), 118.7$ (CH), 148.5 (C), 155.1 (C), 168.0 (C); IR $\left(v_{\max } / \mathrm{cm}^{-1}\right) 3429$, 2976, 2932, 2873, 1745, 1664, 1608, 1362, 1341, 1310, 1220, 1135, 1037; HRMS found $186.1129\left[\mathrm{M}+\mathrm{NH}_{4}^{+}\right], \mathrm{C}_{9} \mathrm{H}_{16} \mathrm{NO}_{3}$ requires 186.1125 .

\section{Compound 23}

To a stirred solution of 22 (17 $\mathrm{mg}, 0.1 \mathrm{mmol}, 1$ equiv) in $\mathrm{CH}_{2} \mathrm{Cl}_{2}(0.5 \mathrm{~mL})$ was added at room temperature imidazole (34 mg, $0.5 \mathrm{mmol}, 5$ equiv) followed by $\mathrm{iPr}_{2} \mathrm{SiCl}_{2}(17.4 \mu \mathrm{L}$, 0.1 mmol, 1 equiv) after complete dissolution of imidazole. After disappearance of 22 (2 hours), 19 (25 mg, $0.1 \mathrm{mmol}, 1$ equiv) in $\mathrm{CH}_{2} \mathrm{Cl}_{2}(0.3 \mathrm{~mL})$ was added to the mixture. The reaction was stirred for $15 \mathrm{~min}$, then quenched with a saturated aqueous solution of $\mathrm{NH}_{4} \mathrm{Cl}$. The aqueous layer was extracted with $\mathrm{CH}_{2} \mathrm{Cl}_{2}$. The combined organic phase were dried over $\mathrm{Na}_{2} \mathrm{SO}_{4}$ and concentrated under vacuum. The crude product was purified by flash chromatography (petroleum ether-diethylether $8: 2)$ to give the silicon tethered ( $23 \mathrm{mg}, 43 \%)$. In Schlenk tube, the silicon tethered $(23 \mathrm{mg})$ was heated $\left(55^{\circ} \mathrm{C}\right)$ in degassed $\mathrm{C}_{6} \mathrm{D}_{6}(1 \mathrm{~mL})$ for 4 hours. The solution was then concentrated and purified by flash chromatography (petroleum ether-diethylether $85: 15)$ to give $23(17 \mathrm{mg}, 74 \%)$. mp $161{ }^{\circ} \mathrm{C} ; \delta_{\mathrm{H}}$ (400 MHz, $\left.\mathrm{C}_{6} \mathrm{D}_{6}\right) 0.04\left(3 \mathrm{H}, \mathrm{s}, \mathrm{CH}_{3}\right), 0.34\left(3 \mathrm{H}, \mathrm{s}, \mathrm{CH}_{3}\right), 0.91$ $\left(3 \mathrm{H}\right.$, br s, $\left.\mathrm{CH}_{3}\right), 1.00-1.07\left(7 \mathrm{H}, \mathrm{m}, 2 \times \mathrm{CH}_{3}\right.$ and $\left.\mathrm{CH}\right), 1.03(9 \mathrm{H}$, $\left.\mathrm{s}, 3 \times \mathrm{CH}_{3}\right), 1.15-1.18\left(7 \mathrm{H}, \mathrm{m}, 2 \times \mathrm{CH}_{3}\right.$ and $\left.\left.\mathrm{CH}\right),\right), 1.34(3 \mathrm{H}, \mathrm{s}$, $\left.\mathrm{CH}_{3}\right), 1.73\left(3 \mathrm{H}, \mathrm{s}, \mathrm{CH}_{3}\right), 2.12(1 \mathrm{H}, \mathrm{d}, J=10.5 \mathrm{~Hz}, \mathrm{CH}), 4.85$ $(1 \mathrm{H}, \mathrm{s}, \mathrm{CH}), 5.16(1 \mathrm{H}, \mathrm{d}, J=10.5 \mathrm{~Hz}, \mathrm{CH}), 5.43(1 \mathrm{H}$, br s, CH), $7.18\left(1 \mathrm{H}, \mathrm{t}, J=7.5 \mathrm{~Hz}, \mathrm{CH}_{\mathrm{Ar}}\right), 7.24\left(1 \mathrm{H}, \mathrm{t}, J=7.5 \mathrm{~Hz}, \mathrm{CH}_{\mathrm{Ar}}\right)$, $7.53\left(1 \mathrm{H}, \mathrm{d}, J=7.5 \mathrm{~Hz}, \mathrm{CH}_{\mathrm{Ar}}\right), 7.84\left(1 \mathrm{H}, \mathrm{d}, J=7.5 \mathrm{~Hz}, \mathrm{CH}_{\mathrm{Ar}}\right)$; $\delta_{\mathrm{C}}\left(100 \mathrm{MHz}, \mathrm{CDCl}_{3}\right)-5.02\left(\mathrm{CH}_{3}\right),-4.6\left(\mathrm{CH}_{3}\right), 13.3(\mathrm{CH})$, $13.9\left(\mathrm{CH}_{3}\right), 14.2(\mathrm{CH}), 17.1_{6}\left(\mathrm{CH}_{3}\right), 17.2\left(\mathrm{CH}_{3}\right), 17.4\left(\mathrm{CH}_{3}\right)$, $17.5\left(\mathrm{CH}_{3}\right), 18.5(\mathrm{C}), 26.2\left(4 \times \mathrm{CH}_{3}\right), 31.5\left(\mathrm{CH}_{3}\right), 55.0(\mathrm{CH})$, $68.5(\mathrm{CH}), 71.5(\mathrm{CH}), 76.8(\mathrm{CH}), 90.2(\mathrm{C}), 119.5(\mathrm{CH}), 123.3$ $(\mathrm{CH}), 123.4(\mathrm{CH}), 127.3(\mathrm{CH}), 128.1(\mathrm{CH}), 134.3(\mathrm{C}), 139.5$
(C), 169.5 (C), 171.6 (C); IR $\left(v_{\max } / \mathrm{cm}^{-1}\right)$ 2933, 2891, 2863, $1756,1645,1465,1348,1253,1201,1133,1073,1028,1016$; MS $m / z(\mathrm{ESI}+) 553[\mathrm{M}+\mathrm{Na}]^{+}$; HRMS found 531.2957 $\left[\mathrm{M}+\mathrm{H}^{+}\right], \mathrm{C}_{29} \mathrm{H}_{47} \mathrm{O}_{5} \mathrm{Si}_{2}$ requires 531.2957.

\section{Compound 24}

To a stirred solution of the $\gamma$-butenolide ${ }^{27}(500 \mathrm{mg}, 2.08 \mathrm{mmol}$, 1 equiv) in anhydrous THF $(11 \mathrm{~mL})$ at $0{ }^{\circ} \mathrm{C}$ was added a solution of HF-pyridine ( $76 \mu \mathrm{L}, 70 \%$ in pyridine, $4.17 \mathrm{mmol}, 2$ equiv). The reaction was stirred at room temperature and followed by TLC. After disappearance of the starting material, the reaction was quenched with a saturated aqueous solution of $\mathrm{NaHCO}_{3}$. The aqueous phase was extracted with ether and the combined organics layers were dried over anhydrous $\mathrm{MgSO}_{4}$, filtered and concentrated in vacuo. The resulting crude product was purified by flash chromatography on silica gel (petroleum ether-ethyl acetate $1: 1)$ to give $24(200 \mathrm{mg}, 76 \%)$ as a $9: 1$ mixture of diastereomers. $\delta_{\mathrm{H}}\left(400 \mathrm{MHz}, \mathrm{C}_{6} \mathrm{D}_{6}\right) 4.06(2 \mathrm{H}, \mathrm{d}$, $\left.J=6.8 \mathrm{~Hz}, \mathrm{CH}_{2}\right), 4.72(1 \mathrm{H}, \mathrm{t}, J=6.8 \mathrm{~Hz}, \mathrm{CH}), 5.44(1 \mathrm{H}, \mathrm{d}, J=$ $5.3 \mathrm{~Hz}, \mathrm{CH}), 6.16(1 \mathrm{H}, \mathrm{d}, J=5.3 \mathrm{~Hz}, \mathrm{CH}) ; \delta_{\mathrm{C}}\left(100 \mathrm{MHz}, \mathrm{C}_{6} \mathrm{D}_{6}\right)$ $57.2\left(\mathrm{CH}_{2}\right), 114.2(\mathrm{CH}), 120.1(\mathrm{CH}), 143.1(\mathrm{C}), 149.2(\mathrm{C})$, 168.8 (C); IR $\left(v_{\max } / \mathrm{cm}^{-1}\right) 3347,2954,2922,2854,1774,1747$, 1677, 1463, 1118, 1065; HRMS found 127.0389 [M $+\mathrm{H}]^{+}$, $\mathrm{C}_{6} \mathrm{H}_{7} \mathrm{O}_{3}$ requires 127.0390 .

\section{Compound 25}

To a stirred solution of $\mathrm{CH}_{2} \mathrm{Cl}_{2}(1.6 \mathrm{~mL})$, imidazole (108 mg, $2.55 \mathrm{mmol}, 5$ equiv) and $\mathrm{PPr}_{2} \mathrm{SiCl}_{2}(58 \mu \mathrm{L}, 0.32 \mathrm{mmol}, 1$ equiv $)$ was added dropwise and at room temperature $19(80 \mathrm{mg}$, $0.32 \mathrm{mmol}, 1$ equiv) in $\mathrm{CH}_{2} \mathrm{Cl}_{2}(0.5 \mathrm{~mL})$. After disappearance of 19 (5 min), lactone 24 (40 mg, $0.32 \mathrm{mmol}, 1$ equiv) in $\mathrm{CH}_{2} \mathrm{Cl}_{2}$ $(1.5 \mathrm{~mL})$ was added to the mixture. The reaction was stirred for $15 \mathrm{~min}$, then quenched with a saturated aqueous solution of $\mathrm{NH}_{4} \mathrm{Cl}$. The aqueous layer was extracted with $\mathrm{CH}_{2} \mathrm{Cl}_{2}$. The combined organic phase were dried over $\mathrm{Na}_{2} \mathrm{SO}_{4}$ and concentrated under vacuum. The crude product was purified by flash chromatography (petroleum ether-diethylether $8: 2$ ) to give the silicon tethered (109 mg, 70\%). In Schlenk tube, the silicon tethered $(109 \mathrm{mg})$ was heated $\left(55^{\circ} \mathrm{C}\right)$ in degassed $\mathrm{C}_{6} \mathrm{D}_{6}(6 \mathrm{~mL})$ for 4 hours. The solution was then concentrated and purified by flash chromatography (petroleum ether-diethylether $85: 15$ ) to give 25 (44 mg, 40\%). $\delta_{\mathrm{H}}\left(400 \mathrm{MHz}, \mathrm{C}_{6} \mathrm{D}_{6}\right)-0.17(3 \mathrm{H}, \mathrm{s}$, $\left.\mathrm{CH}_{3}\right), 0.01\left(3 \mathrm{H}, \mathrm{s}, \mathrm{CH}_{3}\right), 0.87\left(9 \mathrm{H}, \mathrm{s}, 3 \times \mathrm{CH}_{3}\right), 1.08-1.12$ $\left(14 \mathrm{H}, \mathrm{m}, 4 \times \mathrm{CH}_{3}\right.$ and $\left.2 \times \mathrm{CH}\right), 2.63(1 \mathrm{H}, \mathrm{td}, J=10$ and $4 \mathrm{~Hz}$, $\mathrm{CH}), 3.84\left(1 \mathrm{H}, \mathrm{dd}, J=11\right.$ and $\left.4 \mathrm{~Hz}, \mathrm{CH}_{2}\right), 4.01-4.07(1 \mathrm{H}, \mathrm{m}$, $\left.\mathrm{CH}_{2}\right), 4.43(1 \mathrm{H}, \mathrm{s}, \mathrm{CH}), 5.24(1 \mathrm{H}, \mathrm{d}, J=10 \mathrm{~Hz}, \mathrm{CH}), 5.33(1 \mathrm{H}$, $\mathrm{d}, J=5.5 \mathrm{~Hz}, \mathrm{CH}), 5.33(1 \mathrm{H}, \mathrm{d}, J=5.5 \mathrm{~Hz}, \mathrm{CH}), 7.10-7.24$ $\left(3 \mathrm{H}, \mathrm{m}, 3 \times \mathrm{CH}_{\mathrm{Ar}}\right), 7.82\left(1 \mathrm{H}, \mathrm{d}, J=7.5 \mathrm{~Hz}, \mathrm{CH}_{\mathrm{Ar}}\right) ; \delta_{\mathrm{C}}$ $\left(100 \mathrm{MHz}, \mathrm{CDCl}_{3}\right)-4.5\left(\mathrm{CH}_{3}\right),-4.2\left(\mathrm{CH}_{3}\right), 12.4(\mathrm{CH}), 13.6$ (CH), $16.9\left(\mathrm{CH}_{3}\right), 17.0\left(\mathrm{CH}_{3}\right), 17.2\left(\mathrm{CH}_{3}\right), 17.2_{4}\left(\mathrm{CH}_{3}\right), 18.3$ (C), $25.9\left(3 \times \mathrm{CH}_{3}\right), 44.2(\mathrm{CH}), 64.1\left(\mathrm{CH}_{2}\right), 72.3(\mathrm{CH}), 73.8$ $(\mathrm{CH}), 89.8(\mathrm{C}), 121.8(\mathrm{CH}), 125.6(\mathrm{CH}), 127.4(\mathrm{CH}), 127.5$ $(\mathrm{CH}), 128.9(\mathrm{CH}), 134.3(\mathrm{C}), 139.1(\mathrm{C}), 156.7(\mathrm{CH}), 171.9(\mathrm{C})$; IR $\left(v_{\max } / \mathrm{cm}^{-1}\right) 2953,2928,2896,2860,1767,1463,1254$, 1134, 1081, 1027; MS m/z (ESI+) $511[\mathrm{M}+\mathrm{Na}]^{+}$; HRMS found 506.2751 [M $\left.+\mathrm{NH}_{4}^{+}\right], \mathrm{C}_{26} \mathrm{H}_{44} \mathrm{NO}_{5} \mathrm{Si}_{2}$ requires 506.2753. 


\section{Acknowledgements}

The Agence Nationale pour la Recherche (A.N.R.-09JCJC-0036), the CNRS and Aix-Marseille Université (UMR 7313) are gratefully acknowledged for financial support. The authors wish to thank Michel Giorgi for X-Ray analysis. Dr Gaëlle Chouraqui is also gratefully thanked for her fruitful participation.

\section{Notes and references}

1 (a) R. Pradhan, M. Patra, A. K. Behera, B. K. Mishra and R. K. Behera, Tetrahedron, 2006, 62, 779-828; (b) I. Denissova and L. Barriault, Tetrahedron, 2003, 59, 10105-10146.

2 (a) B. Bister, D. Bischoff, M. Strobele, J. Riedlinger, A. Reicke, F. Wolter, A. T. Bull, H. Zahner, H. P. Fiedler and R. D. Süssmuth, Angew. Chem., Int. Ed., 2004, 43, 2574-2576; (b) J. Riedlinger, A. Reicke, H. Zahner, B. Krismer, A. T. Bull, L. A. Maldonado, A. C. Ward, M. Goodfellow, B. Bister, D. Bischoff, R. D. Süssmuth and H. P. Fiedler, J. Antibiot., 2004, 57, 271-279.

3 (a) N. Matsumoto, T. Tsuchida, M. Maruyama, R. Sawa, N. Kinoshita, Y. Homma, Y. Takahashi, H. Iinuma, H. Naganawa, T. Sawa, M. Hamada and T. Takeuchi, J. Antibiot., 1996, 49, 953-954; (b) N. Matsumoto, T. Tsuchida, H. Nakamura, R. Sawa, Y. Takahashi, H. Naganawa, H. Iinuma, T. Sawa, T. Takeuchi and M. Shiro, J. Antibiot., 1999, 52, 276-280; (c) A. Holtzel, A. Dieter, G. Schmid Dietmar, R. Brown, M. Goodfellow, W. Beil, G. Jung and H.-P. Fiedler, J. Antibiot., 2003, 56, 1058-1061; (d) N. Matsumoto, T. Tsuchida, M. Maruyama, N. Kinoshita, Y. Homma, H. Iinuma, T. Sawa, M. Hamada and T. Takeuchi, J. Antibiot., 1999, 52, 269-275.

4 (a) G. Metha and S. Kotha, Tetrahedron, 2001, 57, 625-659; (b) J. L. Segura and N. Martin, Chem. Rev., 1999, 99, 3199-3246; (c) A. K. Sadana, R. K. Saini and W. E. Billups, Chem. Rev., 2003, 103, 15391602; (d) W. Choy, Tetrahedron, 1990, 46, 2281-2286.

5 A. Bartoli, F. Rodier, L. Commeiras, J.-L. Parrain and G. Chouraqui, Nat. Prod. Rep., 2011, 28, 763-782 and see references therein.

6 R. Blanc, V. Héran, R. Rahmani, L. Commeiras and J.-L. Parrain, Org. Biomol. Chem., 2010, 8, 5490-5494.

7 (a) R. M. Ortuño, J. Corbera and J. Font, Tetrahedron Lett., 1986, 27, 1081-1084; (b) D. Alonso, V. Branchadell, J. Font, A. Oliva, R. M. Ortuño and F. Sanchez-Ferrando, Tetrahedron, 1990, 46, 4371-4378; (c) D. Alonso, J. Orti, V. Branchadell, A. Oliva, R. M. Ortuño, J. Bertran and J. Font, J. Org. Chem., 1990, 55, 3060-3063; (d) D. Alonso, J. Font, M. M. Ortuño, J. d'Angelo, A. Guingant and C. Bois, Tetrahedron, 1991, 47, 5895-5900; (e) V. Branchadell, J. Font, A. Oliva, J. Orti, R. M. Ortuño, S. Rafel, N. Terris and M. Ventura, Tetrahedron, 1991, 47, 8775-8786; $(f)$ V. Branchadell, J. Orti, R. M. Ortuño, A. Oliva, J. Font, J. Bertran and J. J. Dannenberg, J. Org. Chem., 1991, 56, 2190-2193; (g) D. Alonso, J. Font and R. M. Ortuño, J. Org. Chem., 1991, 56, 55675572; (h) C. O. de Echaguen and R. M. Ortuño, Tetrahedron Lett., 1995, 36, 749-752; (i) V. Branchadell, J. Font, A. G. Moglioni, C. O . de Echagueen, A. Oliva, R. M. Ortuño, J. Veciana and J. Vidal-Gancedo, J. Am. Chem. Soc., 1997, 119, 9992-10003.

8 S. Rafel, G. Cabarrocas, M. Ventura and T. Parella, J. Chem. Soc., Perkin Trans. 1, 1998, 3837-3843.

9 A. J. Poss and M. H. Brodowski, Tetrahedron Lett., 1989, 30, 25052508.
10 (a) J. Uenishi, R. Kawahama and O. Yonemitsu, J. Org. Chem., 1997, 62, 1691-1701; (b) K. Takeda, M. Sato and E. Yoshii, Tetrahedron Lett., 1986, 27, 3903-3906.

11 K. Takeda, S. Yano, M. Sato and E. Yoshii, J. Org. Chem., 1987, 52, 4135-4137.

12 (a) K. Okumura, K. Okazaki, K. Takeda and E. Yoshii, Tetrahedron Lett., 1989, 30, 2233-2236; (b) K. Takeda, Y. Igarashi, K. Okazaki, E. Yoshii and K. Yamaguchi, J. Org. Chem., 1990, 55, 3431-3434.

13 (a) C. W. Zapf, B. A. Harrison, C. Drahl and E. J. Sorensen, Angew. Chem., Int. Ed., 2005, 44, 6533-6537; (b) B. B. Snider and Y. Zou, Org. Lett., 2005, 7, 4939-4941; (c) E. A. Couladouros, E. A. Bouzas and A. D. Magos, Tetrahedron, 2006, 62, 5272-5279.

14 M. Planas, C. Segura, M. Ventura and R. M. Ortuño, Synth. Commun., 1994, 24, 651-654.

15 (a) C. Cox and S. J. Danishefsky, Org. Lett., 2000, 2, 3493-3496; (b) C. Cox and S. J. Danishefsky, Org. Lett., 2001, 3, 2899-2902; (c) J. P. Deville and V. Behar, Org. Lett., 2002, 4, 1403-1405; (d) T. R. Kelly, D. Xu, G. Martinez and H. Wang, Org. Lett., 2002, 4, 1527-1529; (e) C. D. Cox, T. Siu and S. J. Danishefsky, Angew. Chem., Int. Ed., 2003, 42, 5625-5629; $(f)$ T. R. Kelly, X. Cai, B. Tu, E. L. Elliott, G. Grossmann and P. Laurent, Org. Lett., 2004, 6, 4953-4956; $(g)$ D. A. Henderson, P. N. Collier, G. Pave, P. Rzepa, A. J. P. White, J. N. Burrows and A. G. M. Barrett, J. Org. Chem., 2006, 71, 2434-2444; (h) V. Le, A. J. P. White, J. N. Burrows and A. G. M. Barrett, Tetrahedron, 2006, 62, 12252-12263; (i) P. J. Parsons, J. Board, A. J. Waters, P. B. Hitchcock, F. Wakenhut and D. S. Walter, Synlett, 2006, 3243-3246; (j) H. Wehlan, E. Jezek, N. Lebrasseur, G. Pave, E. Roulland, A. J. P. White, J. N. Burrows and A. G. M. Barrett, J. Org. Chem., 2006, 71, 8151-8158; (k) P. J. Parsons, A. J. Waters, D. S. Walter and J. Board, J. Org. Chem., 2007, 72, 1395-1398; (l) P. J. Parsons, J. Board, D. Faggiani, P. B. Hitchcock, L. Preece and A. J. Waters, Tetrahedron, 2010, 66, 6526-6533; $(m)$ K. Watanabe, Y. Iwata, S. Adachi, T. Nishikawa, Y. Yoshida, S. Kameda, M. Ide, Y. Saikawa and M. Nakata, J. Org. Chem., 2010, 75, 5573-5579; (n) S. A. Jacques, B. H. Patel and A. G. M. Barrett, Tetrahedron Lett., 2011, 52, 6072-6075; (o) S. A. Jacques, S. Michaelis, B. Gebhardt, A. Blum, N. Lebrasseur, I. Larrosa, A. J. P. White and A. G. M. Barrett, Eur. J. Org. Chem., 2012, $107-113$.

16 K. Tatsuta, H. Tanaka, H. Tsukagoshi, T. Kashima and S. Hosokawa, Tetrahedron Lett., 2010, 51, 5546-5549.

17 T. Siu, C. D. Cox and S. J. Danishefsky, Angew. Chem., Int. Ed., 2003, 42, 5629-5634.

18 A. Bartoli, G. Chouraqui and J.-L. Parrain, Org. Lett., 2012, 14, 122 125.

19 (a) T. Momose, N. Toyooka and Y. Takeuchi, Heterocycles, 1986, 24, 1429-1431; (b) S. Gelin and P. Pollet, Synth. Commun., 1980, 10, $805-812$.

20 A. B. Smith III and R. J. Fox, Org. Lett., 2004, 6, 1477-1480.

21 M. S. South and L. S. Liebeskind, J. Org. Chem., 1982, 47, 3815-3821.

22 J. G. Allen, M. F. Hentemann and S. J. Danishefsky, J. Am. Chem. Soc., 2000, 122, 571-573.

23 L. Fensterbank, M. Malacria and S. McN. Sieburth, Synthesis, 1997, $813-854$.

2420 was obtained by treatment of the corresponding OTBS protected lactone with HF.Pyridine.

25 I. Paterson and T. Temal-Laïb, Org. Lett., 2002, 4, 2473-2476.

26 CCDC 849361 contains the supplementary crystallographic data for this paper.

27 S. Inack-Ngi, R. Rahmani, L. Commeiras, G. Chouraqui, J. Thibonnet, A. Duchêne, M. Abarbri and J.-L. Parrain, Adv. Synth. Catal., 2009, 351, 779-788. 